\title{
Quantum Biology: Does quantum physics hold the key to revolutioniz- ing medicine?
}

\author{
Bey Hing Goh ${ }^{1,2^{*}}$, Eng Siang Tong ${ }^{3,4}$, Priyia Pusparajah ${ }^{5 *}$ \\ ${ }^{1}$ College of Pharmaceutical Sciences, Zhejiang University, 866 Yuhangtang Road, Hangzhou 310058, China \\ ${ }^{2}$ Biofunctional Molecule Exploratory (BMEX) Research Group, School of Pharmacy, Monash University Malaysia, 47500 \\ Bandar Sunway, Selangor Darul Ehsan, Malaysia. \\ ${ }^{3}$ Department of Social and Preventive Medicine, Institute of Postgraduate Studies, Wisma Research and Development \\ University Malaya, University Malaya, 50603 Kuala Lumpur \\ ${ }^{4}$ Geopathology Research Institute, Singapore, 14 Robinson Road, \#08-01A Far East Finance Building, Singapore 048545. \\ ${ }^{5}$ Medical Health and Translational Research Group, Jeffrey Cheah School of Medicine and Health Sciences, Monash \\ University Malaysia, 47500 Bandar Sunway, Selangor Darul Ehsan, Malaysia
}

\begin{abstract}
Quantum biology is the study of quantum effects on biochemical mechanisms and biological function. Quantum physics describes the behaviour of the nanoscale particles that make up all matter including living organisms, but it was generally believed that the 'warm, wet and noisy environment' in living organisms would result in decoherence making it impossible for meaningful quantum effects to occur. However, there is now a substantial body of work supporting that nature has actually adapted in such a way as to exploit quantum properties to enhance cellular functioning and is believed to have role in a diverse range of key processes in living organisms ranging from maintaining the stability of DNA to neuron function to conscious cognition; from light harvesting in photosynthesis to avian magnetic field based navigation. This review aims to summarize the various mechanisms and functions in living organisms believed to utilize quantum mechanics to purposefully and effectively enhance their performance, and to explore the potential this could hold in diagnosing and treating various medical conditions.
\end{abstract}

Keywords: quantum; revolution; medicine; photosynthesis; radiation; heath; biology

Received: $9^{\text {th }}$ October 2020

Accepted: $29^{\text {th }}$ October 2020

Published Online: $10^{\text {th }}$ November 2020
*Correspondence: Bey Hing Goh, School of Pharmacy, Monash University Malaysia; goh.bey.hing@monash.edu; Priyia Pusparajah, Jeffrey Cheah School of Medicine and Health Sciences, Monash University Malaysia, Bandar Sunway, Subang Jaya, Selangor, Malaysia; priyia.pusparajah@monash.edu

Citation: Goh BH, Tong ES, and Pusparajah P. Quantum Biology: Does quantum physics hold the key to revolutionizing medicine?. Prog Drug Discov Biomed Sci 2020; 3(1): a0000130. https://doi.org/10.36877/pddbs.a0000130.

\section{Background}

Quantum physics is the branch of physics that deals with the domain of the very small - at the scale of individual atoms and their particles ${ }^{[1,2]}$; seeking to understand why tiny particles like atoms, photons and electrons behave in ways so unlike objects on the scale of basketballs, butterflies and bacteria. Although quantum phenomena seem complex and unpredictable, they can be expressed as mathematical equations and laws that govern and predict the behavior on particles ${ }^{[3,4]}$. Biology, on the other hand is the science of life itself, consisting of organisms at a scale far beyond that thought to be relevant to quantum mechanics; instead being assumed to operate based only on the laws of classical Newtonian physics.

On one hand, given the fact that all living organisms are at their heart made up of atoms which in turn operate on the laws of quantum physics, it seems only logical that quantum physics should have a role in explaining life. However, given that quantum phenomena were only able to be replicated at detectable levels for a meaningful duration at the nanometer scale, in a vacuum, at ultralow temperatures, it was presumed that the warm, wet and noisy environment in living beings would not be capable of supporting quantum mechanic ${ }^{[1,2,5]}$. However, in recent years, evidence has emerged from a variety of experiments across a range of processes and functions in living organisms that seem to be best explained by quantum mechanics rather than classical physics ${ }^{[1,2,6-8]}$; which have led to the emergence of the fascinating new field of quantum biology.

Quantum biology is the study of quantum effects on biochemical mechanisms and biological function ${ }^{[9]}$. While biology and physics may seem to many to be a very unlikely partnership, the fathers of quantum physics had hinted at their anticipation that links between the two 
would be found. In the last 2 decades there have been significant findings showing that quantum mechanics do play a role in key processes in living organisms including the organization of information in $\mathrm{DNA}^{[10,11]}$, photon capture in photosynthesis ${ }^{[12]}$ and vision ${ }^{[13,14]}$, enzyme catalysis particularly in the mitochondria ${ }^{[9]}$, avian navigation ${ }^{[15]}$ and olfaction ${ }^{[7]}$. While there are still many arguments questioning the genuine significance of these findings, the experimental evidence supporting the existence of these processes at a detectable and meaningful level in living organisms is strong enough to have begun to revolutionize the way scientists perceive life - some of which we will discuss in this article. It seems to be increasingly accepted that quantum mechanics can apply in a variety of conditions that were not thought to be possible a few decades ago ${ }^{[5,12,16-18]}$ and a burning question now is actually what the cut off is between quantum and classical mechanics, and for biologist's and clinicians, this then leads to asking how this could provide new insights into life, health and disease.

If nature has indeed cracked the code of how to utilize quantum mechanics in the chaotic, warm environment of a living organism, unravelling how this is possible holds tremendous implications across a variety of fields including quantum computing and other machinery; and, for many, there is hope that these developments may herald a new era in clinical medicine. If a multitude of core processes in the human body are indeed meaningfully utilizing quantum mechanics, this opens up a whole new vista of novel approaches to diagnosis and management of a range of diseases including seemingly insurmountable health challenges like cancer.

\section{Key concepts in quantum physics}

Quantum physics is widely known for its weirdness. One of the most well-known examples illustrating just how weird things can get in the quantum world is Schrödinger's cat, a thought experiment devised by Erwin Schrödinger in $1935^{[19]}$. Schrodinger imagined a cat being placed in a box together with a potentially lethal radioactive substance; and stated that until the box was opened, the cat was actually possibly both dead or alive, and would be in this superposition of potentially being both until the box was opened and the cat observed. As bizarre and hard to accept as this may sound, the capability of one object to be in multiple states at the same time as been validated experimentally on the quantum scale ${ }^{[20]}$.

The entire discipline of quantum physics operated for many years on the tenet that quantum mechanics could only apply at the nanometer level, which seemed to be a logical conclusion based on two key elements of quantum theory namely Planck's constant and De Broglie's equation $^{[3]}$. Maxwell Planck made the first step in quantum physics when he discovered that energy, which had always been assumed to be emitted in a continuous spectrum, was released from atoms in 'quantums' or discrete packets of energy. While measuring energy emitted from heated objects, he found that the energy released formed distinct peaks rather than a continuous spectrum; and these were all multiples of a minimum value which is now known as the Planck constant $\left(\mathrm{h}=6.626 \times 10^{-37} \mathrm{~J} . \mathrm{s}\right)^{[21]}$. This was the first suggestion that waves could also demonstrate particle like properties - a phenomena which is now known as wave-particle duality which forms the very fabric of quantum theory. The Planck constant's role in quantum theory is comparable to the role of the speed of light in relativity theory - telling us when quantum effects will be important; with the very small value of the Planck constant suggesting that quantum phenomena were only observed in very small particles $^{[22]}$.

Louis De Broglie later proposed the then radical idea that if waves could have particle like properties, matter should also have wave like properties ${ }^{[23,24]}$. This was initially greeted with skepticism but was eventually conclusively demonstrated by means of the demonstrating that particles were able to generate measurable interference (a key property of waves) using Young's double slit experiment ${ }^{[24]}$. This was first demonstrated using electrons to generate interference patterns, proving that particles could indeed behave like waves. In yet another twist in the quantum tale, however, it turned out that the interaction of an observer in the system (by observing which slit the electron went through) then resulted in the loss of interference pattern - meaning if one observed the particle like behavior, one would then be unable to detect the wave behavior ${ }^{[22]}$.

De Broglie's equation, stated that matter wave lengths were a function of the Planck constant and the momentum of the particle, $\lambda=\mathrm{h} / \mathrm{p}=\mathrm{h} / \mathrm{mv}^{[25]}$. The wavelength of large objects is extremely small, for example a tennis ball would have a wave length of approximately $10^{-34} \mathrm{~m}$, while that of an electron is $1.2 \mathrm{~nm}$. In order for waves to interact, they need to have similar wavelengths, and the frequency of matter waves for macroscopic objects is so miniscule that there is no object able to effectively interact thus there is a loss of quantum mechanics in in larger objects. It is worth noting the De Broglie equation does not apply to photons as they have no mass ${ }^{[26]}$.

The fact that quantum phenomena seemed to only apply at the nanometer scale seemed to make it almost impossible to conceive that these could possibly occur at the scale of a living organism. The fact that Planck's constant is so small seems to suggest that the wavelengths of large objects are far too small to have detectable interactions ${ }^{[22]}$. However, more and more scientists are seriously beginning to question whether large objects, like cats, can be demonstrated to show some of the strange quantum features that are now well known in atoms and particles; and their results are showing that there is no clear cut boundary where quantum effects end and classical physics takes over ${ }^{[27]}$. In theory, it is perfectly possible to design experiments where massive objects behave as if they were atoms in at least one degree of freedom; but in reality, it is virtually impossible to isolate large objects from their environment. This is because macroscopic objects constantly interact through exchange of photons, which results in heating and therefore decoherence ${ }^{[28,29]}$; with decoherence here referring to the disruption of the constant phase relationship necessary for quantum states; however nature evidently has a few tricks up her sleeve 
and some key findings that have made the notion of quantum biology more believable include the findings that quantum effects can be seen to be operating at room temperature ${ }^{[6]}$, and that quantum phenomena can exist at a macroscopic level with Arndt and his team having been able to demonstrate interference through the interaction of particle in excess of $2 \mathrm{kDalton}^{[30]}$. In fact Malvalava and her team recently reported observing that quantum fluctuations were able to produce measurable movement, albeit of a tiny degree, of the 40 kilogram mirrors of the U.S National Science Foundation's Laser Interferometer Gravitational-wave Observatory (LIGO) as the result of quantum fluctuations ${ }^{[31]}$

Zurek has proposed a slight variation of these views and states that the perception of objective classical reality actually arises from the quantum substrate ${ }^{[29,32]}$. This is proposed to be the result of a process he calls environmentinduced superselection -or einselection for short. While it has always been a principle of quantum physics that interaction with the environment results in loss of predicted quantum behaviour, einselection proposes that the observed decoherence in open quantum systems that are interacting with their environment, is because only preferred pointer states that can remain stable in spite being immersed in the environment are selected; thus the superpositions, which are inherently unstable, quickly decay into a mixture of einselected, stable pointer states, which we observe as our familiar classical reality ${ }^{[4,32]}$. To quote Zurek, "the effective amplification they represent accounts for the objective existence of the einselected states of macroscopic quantum systems though the redundancy of pointer state records in their environment through quantum Darwinism"[32].

A full review of the theory of quantum physics is beyond the scope of this paper, but in order to fully appreciate the involvement of quantum physics in the realm of biology, we aim to provide a brief description of some of the fundamental concepts involved in quantum mechanics. At the root of quantum physics are the concepts of wave-particle duality, non-locality, superposition, entanglement, and tunnel effect. It can be said that any research where the Planck constant and any or all of these characteristics are mentioned belongs to the realm of quantum physics ${ }^{[33]}$.

As mentioned earlier, one hallmark of particles demonstrating wave like characteristics is the production of interference. This results from waves summating to either increase or decrease their amplitude. Coherence, a term often used in quantum mechanics, refers to well defined phase and amplitude relations where correlations are preserved over separations in time or space, essentially meaning that 2 waves are in sync with each other. Coherence must be present before the particles can display many of the hallmark properties of quantum physics ${ }^{[34,35]}$. However, quantum coherence becomes increasingly more difficult to maintain as molecules become larger and more complex. Environmental interaction and vibrational modes have a tendency to cause decoherence of quantum superpositions. Given that living organisms consist of large and complex molecules, most biomolecular mechanisms have essentially been modeled as classical processes ${ }^{[36]}$, as it was very uncertain whether the relevant degrees of freedom within cells and tissues could be isolated to a sufficient degree to retain their quantum coherence ${ }^{[37]}$.

However, new work has suggested that coherent backscattering and quantum beating may be adaptations life has made to preserve coherence under challenging conditions [5, 38, 39]. An example of coherent backscattering occurs in Saturn's rings where sunlight falling on the millions of ice crystals actually gets reflected in a precise configuration which allows their amplitudes to perfectly sum up; this makes the rings appear much brighter when the Sun is aligned along the direction of Earth from Saturn ${ }^{[40-42]}$. Adding wave amplitudes seems to be able to give rise to more potent effects than might have been anticipated, supporting the idea that some objects in nature have a design that confers the ability to 'harness' coherence, possibly allowing for functional benefits. As mentioned earlier, heating an object tends to result in decoherence, but the phenomenon termed 'quantum beating' has been observed experimentally, with reference to the ability to use thermal vibrations to 'pump' coherence, rather than disrupt it. These coherence phenomena have been demonstrated in various biological and materials systems, suggesting the viability of coherence-enhanced function; with coherence currently being discussed as a means of improving transport in complex, disordered systems ${ }^{[40]}$. Coherent superposition of electronic states has been detected in the light harvesting complexes of photosynthetic bacteria, which means that the energy in light can be very efficiently harvested and transferred using wavelike resonance ${ }^{[43]}$.

Superposition is a property of quantum particles to be in multiple places simultaneously within a zone of probability. This translates to a particle simultaneously occupying all possible locations, a property that is highly sought after to mimic in quantum computing as it would allow simultaneously testing of all possible outcomes. As odd as it sounds, this characteristic has been proven experimentally with a single photon fired at a double slit being able to interfere with itself ${ }^{[44,45]}$. However, the quirky nature of photons was demonstrated when an attempt to actually detect the photon itself passing through the slit resulted in the loss to its ability to create interference. Interaction with the environment, including observation of a particular facet of a particular particle will result in the loss of the other quantum properties which are also known to exist ${ }^{[46]}$. In living organisms, superposition appears to have roles in optimizing the efficacy of light capture in photosynthesis ${ }^{[12,47]}$ and possibly in mutagenesis in $\mathrm{DNA}^{[8,10]}$.

Although there is now little doubt superposition exists, many people have questioned how long this superposition can actually survive - with the key question being whether it can last long enough to have functional value ${ }^{[29]}$ ? One way to try to calculate this involves using the results of previous analysis which yielded the relationship that the ratio of decoherence time to dissipation time is $\mathrm{h}^{2} /\left(\mathrm{mx}^{2} \mathrm{kT}\right)$, where $\mathrm{m}$ is the mass of the molecule, $\mathrm{x}$, is the coherence length (a few nanometers and comparable to the molecular size), and $\mathrm{T}$ is the temperature, ,300 K. If typical values 
of standard environmental conditions are plugged into this equation, the dissipation is in the range of seconds to milliseconds, which in turn would lead to decoherence times of nano- to pico-seconds. This suggests that only processes on similar or shorter timescales can survive interaction with the environmental interaction and persist long enough before experiencing decoherence to be able to affect the dynamics of a system ${ }^{[27]}$. Although this a very short time, biologically meaningful processes, such as processes involved in photosynthesis do occur at the femto to pico second scale, and there do seem to be adaptations in living cells that enhance quantum behaviour, though their exact nature remains to be determined.

Entanglement is a property of 2 coherent particles such that they have the ability of to 'know' the state of the other when one is observed, instantaneously, regardless of distance ${ }^{[48]}$. This is the phenomenon famously dubbed somewhat derisively by Einstein as 'spooky action at a distance' as Einstein was initially unconvinced that the information between the particles could travel faster than light ${ }^{[49,50]}$. However, a series of robust experiments has clearly demonstrated this to be true, however counterintuitive it may seem. With John Bell's work in $1964^{[51]}$ preparing the theoretical ground and Alain Aspect ${ }^{[52]}$ confirming entanglement experimentally. This was demonstrated recently when two entangled photons were sent along very different routes towards an identical end target and both still reached at the same time ${ }^{[53]}$. Recently, satellite-based entanglement distribution to receiver stations separated by more than $1200 \mathrm{~km}$ was demonstrated, with these results being heralded as holding promise for the possibility of a future global quantum communication network ${ }^{[17]}$. Vedral et al. have also been able to demonstrate entanglement in macroscopic matter ${ }^{[27]}$. It does seem to appear that there is no "cutoff point" demarcating the boundary between classical physics and quantum physics ${ }^{[2,54]}$. After all, all objects are in a state of entanglement with each other, which is simply a characteristic of matter at the atomic scale. One property often discussed in relation to entanglement is quantum spin, the property of elementary particles ${ }^{[55]}$ that determines their behaviour in a magnetic field.

'Tunnelling' is a process where a particle can metaphorically 'tunnel 'through a potential-energy barrier and appear on the other side, even though it did not possess sufficient kinetic energy to surmount that barrier. This sort of behaviour cannot be explained by classical mechanics but makes sense in quantum physics, as it can be thought of as a manifestation of the wave-nature of particles. As mentioned earlier, the lighter a particle, the larger its wavelength. Electrons, in particular, are very light compared to atoms, and have wavelengths as large as or larger than atoms at energies found in the valence shells of molecules. Thus, they easily ooze through and around atoms and molecules. However, this behaviour is not limited to electrons; heavy particles such as nuclei, atoms, molecules have also demonstrated tunnelling ${ }^{[56]}$. The phenomenon of tunnelling seems to have a role to play in enzyme catalysis, as closer measurements of the dynamics of enzymatic reactions do not seem to fully explained by classical theories ${ }^{[5,57]}$. In addition, tunnelling is thought to have a role in adaptive mutations ${ }^{[8]}$.

While all these behaviours are typically described individually, they are all aspects of every quantum system, and they are all interlinked, with entanglement only being able to manifest among coherent particles; entanglement also stems from superposition which in turn is what lies at the heart of coherence. A clear link between coherence and entanglement was clearly shown by Adesso and his team both in quantitative and operational terms; demonstrating that all these elements of quantum theory are interlinked ${ }^{[58]}$.

\section{Quantum biology phenomena}

Quantum biology is defined as the application of the features of quantum physics to biological objects and structures ${ }^{[54]}$. There seem to be two possible angles this can be viewed - the first being that since all chemical processes - which are the essence of life - are at their core quantum physical since they manipulate properties at the nanoscale level including ionisation and formation of chemical bonds; and the second being that it is virtually impossible for quantum mechanics to function at a meaningful level at a macroscopic scale in a warm, wet and noisy environment of a living organism that would appear to be a definite recipe for decoherence. Many people take the latter view, after all, no one has ever actually witnessed macroscopic objects such as a cat, a person or a planet in superposition or a state of quantum entanglement. Additionally, there is the argument that even though living cells are made up of fundamental particles and as such should be described by quantum rather than classical mechanics, the quantum effects should cancel each other out in the chaotic environment inside a cell. A fascinating question scientists are currently asking is whether or not quantum phenomena such as entanglement, coherence and tunnelling might play a nontrivial role in enhancing the efficacy of biological processes $^{[55]}$; and there are scientists who already state with confidence that they $\mathrm{do}^{[30]}$.

These ideas have led scientists to question where exactly the microscopic and macroscopic worlds cross over and if the strangest aspects of the quantum world could truly influence living things? For the past two decades the emerging field of quantum biology has been seeking answers for such questions, proposing and performing experiments on living organisms that aim to explore how far we can push the current notions of the limits of quantum theory.

The overlap between physics and biology, while often unexpected, was actually hinted at right at the dawn of quantum physics by the founding fathers of quantum physics including Erwin Schrodinger himself who wrote a book entitled 'What is Life?' in 1945. Schrodinger was the first physicist to deal with quantum biology, and its extension, the DNA structure. Another key figure in the history of quantum biology is Herbert Fröhlich — the physicist who proposed that coherence similar to the BoseEinstein condensate, which is one of the basic features of quantum physics -could exist in biological systems and cells. One idea he argued for strongly was that coherence 
could be the basis of biological oscillators ${ }^{[59]}$, an idea which more recent work suggests was absolutely right.

The results of the work done by quantum biologists clearly support that quantum phenomena do exist in living cells ${ }^{[7,16]}$ but questions remain if these are genuinely representative of a true biological advantage or simply trivial findings. Are living organisms genuinely making use of quantum mechanics to perform tasks that cannot be done classically or to perform tasks more efficiently than even the best classical equivalent? Could some organisms have deliberately adapted their design to harness quantum mechanics in order to gain an edge over their competitors? And do these organisms that exhibit these phenomena have these traits by serendipity or by design?

Given that there is increasing experimental data supporting the existence of meaningful quantum effects in living organisms, it appears that there are unique elements in living cells - as opposed to inanimate systems - that facilitate interactions between fundamental particles and the environment of the cell which allows their macroscopic behaviour to be determined by quantum rather than classical laws ${ }^{[60]}$. The expectation that the 'warm-wet-noisy' environment would not support quantum mechanical phenomena was based on the fact that in inanimate systems such as chemical, heat or electrical engines, their action is governed by the random and incoherent motion of the individual particles whereby decoherence has wiped out the quantum phenomena occurring at the microscopic level. It could be worded differently to say that slight statistical deviations from randomness are actually what result in the macroscopic behaviour of these systems. In living cells however, it appears that their macroscopic behaviour may be determined by the dynamics of individual particles rather than overall motion of the collective particles, and thereby by subject to quantum, rather than statistical laws ${ }^{[8]}$. One of the core conundrums of quantum biology is how some biological systems can remain quantum coherent for a long time at room temperature. That this is possible has been demonstrated by Vattay et al who showed that systems with "the right level of complexity between chaos and regularity can increase their coherence time by orders of magnitude"[60]; however it remains to be unravelled what properties within these systems make this possible.

There are multiple examples of quantum phenomena being detected in living organisms - quantum beating in photosynthetic organisms ${ }^{[6]}$, electron transfer between the bacteria of the same species and with other species via 'bacterial nanowires' which may depend on quantum tunnelling ${ }^{[61]}$, tunnelling in olfaction, quantum entanglement in magnetoreception ${ }^{[62]}$, maintenance of the stability of the double helix structure of $\mathrm{DNA}^{[63]}$ and enzyme catalysis ${ }^{[64]}$; superposition in photosynthesis ${ }^{[12]}$, information processing in the brain ${ }^{[65]}$, adaptive mutation $^{[8]}$ to name a few. From a scientific perspective, it is particularly intriguing how nature has managed to adapt systems in complex living organisms that are able to preserve functional quantum mechanics which previously have only been demonstrated at ultra-low temperature, tightly controlled conditions.

Melkikh and Krennikov concluded from currently available evidence that "the existence of non-trivial quantum effects is necessary for the functioning of living systems; in particular, that classical mechanics cannot explain the stable work of the cell and any over-cell structures"'[66]. Indeed, there have been several key phenomena in living organisms that have demonstrated to utilize quantum phenomena. These are briefly summarized in Table 1, and we will briefly review some of these which have been the subject of more extensive research and are generally well accepted to be examples of quantum physics at work in living organisms.

Table 1 - Summary of biological phenomena involving quantum physics

\begin{tabular}{|c|c|c|}
\hline Biological phenomena & Quantum principles & References \\
\hline \multirow[t]{3}{*}{ DNA stability } & $\begin{array}{l}\text { Quantum properties influencing stability of structure to allow } \\
\text { for fidelity of inheritance of genetic traits across generations. }\end{array}$ & (Rieper, Anders, \& Vedral, 2010; Šponer et al., 2012) \\
\hline & $\begin{array}{l}\text { Tautomerism as the basis for adaptive mutations which may } \\
\text { underlie antibiotic resistance }\end{array}$ & (McFadden \& Al-Khalili, 1999) \\
\hline & $\begin{array}{l}\text { Quantum theory used for developing models potentially } \\
\text { explaining biological and biochemical processes such as the } \\
\text { effects of carcinogens on genes, the mechanism of interactions } \\
\text { of chemotherapy drugs with DNA(Friedman, Boye, \& } \\
\text { Flatmark, 2013) and the understanding of DNA mutations and } \\
\text { defective protein synthesis.(Djordjevic, 2012) }\end{array}$ & $\begin{array}{l}\text { (Friedman et al., 2013), (Djordjevic, 2012; } \\
\text { Uthamacumaran, 2017) }\end{array}$ \\
\hline \multirow[t]{2}{*}{$\begin{array}{l}\text { Oxidative phosphorylation } \\
\text { in mitochondria }\end{array}$} & $\begin{array}{l}\text { Electron transport chain involves quantum biological tunneling } \\
\text { in transfer of electrons }\end{array}$ & (Usselman et al., 2016; Xin et al., 2019) \\
\hline & $\begin{array}{l}\text { Proton transferring complexes utilizing quantum tunneling } \\
\text { occurs in mitochondria as part of processes in enzymes } \\
\text { electron transport, chemiosmosis and catalysis }\end{array}$ & $\begin{array}{l}\text { (Go \& Jones, 2017; Haydon, McGlynn, \& Robus, 2011; } \\
\text { Nunn, Guy, \& Bell, 2016) }\end{array}$ \\
\hline
\end{tabular}




\begin{tabular}{|l|l|l|}
\hline Olfaction & $\begin{array}{l}\text { Olfactory neurons sense vibrational spectra using quantum } \\
\text { tunneling rather than physical shape }\end{array}$ & $\begin{array}{l}\text { (Franco, Turin, Mershin, \& Skoulakis, 2011; Hoehn, } \\
\text { Nichols, Neven, \& Kais, 2015) }\end{array}$ \\
\hline Vision & $\begin{array}{l}\text { Quantum mechanics underlies photoisomerization of retinal } \\
\text { rhodopsin }\end{array}$ & $\begin{array}{l}\text { (Lakhno, Shigaev, Feldman, Nadtochenko, \& Ostrovsky, } \\
\text { 2016; Shigaev, Feldman, Nadtochenko, Ostrovsky, \& } \\
\text { Lakhno, 2020; Sia, Luiten, Stace, Wood, \& Casson, } \\
\text { 2014) }\end{array}$ \\
\cline { 2 - 3 } & $\begin{array}{l}\text { Coherent optical control of retinal isomerization in } \\
\text { bacteriorhodopsin }\end{array}$ & (Nogly et al., 2018; Prokhorenko et al., 2006) \\
\hline Photosynthesis & $\begin{array}{l}\text { Light harvested by antenna transported via FMO complex with } \\
\text { high efficiency due to quantum coherence and superposition }\end{array}$ & (Collini et al., 2010; Engel et al., 2007; Scholes, 2010) \\
\hline Avian Magnetoreception & $\begin{array}{l}\text { Radical pair mechanism utilizing spin characteristics of unpaired } \\
\text { electrons in retina gives rise to ability to use earth's magnetic field for } \\
\text { navigation }\end{array}$ & $\begin{array}{l}\text { (Bandyopadhyay, Paterek, \& Kaszlikowski, 2012; Cai \& Plenio, } \\
\text { 2013; Hiscock et al., 2016) }\end{array}$ \\
\hline Neurobiology & $\begin{array}{l}\text { Quantum entanglement may give rise to firing of neurons; quantum } \\
\text { mechanics better explains generation of action potentials compared to } \\
\text { classical equations }\end{array}$ & $\begin{array}{l}\text { (Bernroider \& Roy, 2005; Bernroider \& Summhammer, 2012; } \\
\text { Khrenikov, Basieva, Pothos, \& Yamato, 2018; Moradi, } \\
\text { Scholkmann, \& Salari, 2015) }\end{array}$ \\
\cline { 2 - 4 } & $\begin{array}{l}\text { Quantum computation occurs in microtubule protein assemblies in the } \\
\text { neurons of the brain and that this results in consciousness }\end{array}$ & $\begin{array}{l}\text { (Adams \& Petruccione, 2020; Baars \& Edelman, 2012; Sultan } \\
\text { Tarlac1, 2011; S. Tarlac1 \& Pregnolato, 2016) }\end{array}$ \\
\hline
\end{tabular}

\section{DNA stability -the basis of heredity}

A breakthrough in biology was the discovery of DNA by Friedrich Miescher in 1860, though its true significance was only realised in the 1950 s after the breakthrough made by Watson and Crick in piecing together the pieces of the puzzle already in place by Levene, Chargaff and Franklin and proposing the double helix structure we are so familiar with today ${ }^{[67]}$. Their work provided a deeper understanding of the incredible simplicity of the basic principles of the genetic code, and current evidence suggests quantum mechanics play a role in maintaining the structure of this molecule at the heart of every living organism. Even before DNA and the details of its structure were known, many remarkable predictions about genetic material were made by one of the fathers of quantum physics, Erwin Schrodinger, who gave 3 public lectures in 1943 on a very unusual topic for a physicist — 'What is Life?'. These lectures were later turned into a book bearing the same name ${ }^{[19]}$.

One of Schrodinger's main aims in his series of talks was to explain how living things seem to defy the second law of thermodynamics which states that all order in the universe tends to break down. Since genetic traits were able to be handed down reliably over multiple generations, this suggested to him that quantum mechanics must be involved. Schrodinger recognized that what we take for granted in terms of the stability of hereditary traits such as a prominent chin being passed from parent to their children, is actually in many ways a violation of many principles of classical physics. One of the elements he questioned was how the portions of our DNA responsible for a particular inherited characteristic could remain 'unperturbed by the disordering tendency of the heat motion for centuries?' ${ }^{[19]}$.

The literature contains a number of published works describing how quantum mechanics have a crucial role in maintaining the stable structure of our genetic code ${ }^{[18,68]}$, in maintaining its stability through numerous cycles of replication, and how it may also have a role in explaining adaptive mutation ${ }^{[8]}$. DNA is composed of nucleic acids which comprise three linked entities: a phosphate group with a deoxyribose sugar and one of four planar bases adenine $(A)$, cytosine $(C)$, guanine $(G)$, or thymine $(T)$. The basic structure of DNA is a double helix made up to 2 chains of nucleic acids, which if unwound would resemble a ladder with its sides made up of the sugar-phosphate backbone and the rungs made up of the bases which are attached to the sugar moiety. The two strands are held together by bonds between the bases, adenine forming a base pair with thymine, and cytosine forming a base pair with guanine; with their specific sequence coding all the genetic information of an organism. Maintaining the structure and order of DNA may actually be best explained by quantum rather classical mechanics. Home and Chattopadhyaya suggested that DNA may actually exist as a "superposition of mutational states"[69]. This proposal supports the idea that living cells are able to retain quantum coherence at room temperature through their ability to maintain an ordered structure compatible with this at temperatures significantly higher than would support such behavour in an inanimate system. Al-Khalili and McFadden have also described how the dynamics of the particles within DNA molecules can result in the development of entanglement. If this theory is true, it is an example of quantum mechanics facilitating interaction between the macroscopic cellular elements and individual particles ${ }^{[8]}$.The stability of the DNA double-helix structure is partly attributable to the fact that since the energy level spacing of the molecules that participate in DNA replication is larger than the thermal and other disturbances, the replication process will be stable ${ }^{[63]}$. The sugar-phosphate backbone, although chemically monotonous and often 
overshadowed by the more exciting base pairs, is in truth the key player which affects every aspect of nucleic acids structure, dynamics, function and evolution. Recently, however, the long underappreciated backbone is now becoming a focus of intense research, and it has been has been shown that its structure and its stability cannot be satisfactorily explained by classical mechanics echoing the thoughts of Schrodinger at the dawn of quantum physics ${ }^{[11]}$. The classical double helix of DNA in many ways has its roots in quantum physics as this structure exists as determined by the allowed bond angles in the sugar-phosphate backbone, the interaction between the two strands and how the hydrophobic bases stack.

The precise arrangement of the particles that make up DNA molecule actually serve to limit the degrees of freedom upon which can be affected by destabilizing forces such as heat; and this is believed to allow for extended temporal coherence of the system. This is believed to create conditions which allow for quantum logic processes to occur - all of which essentially centred around the ability to create and sustain coherence ${ }^{[70]}$.

One method that may simplify the visualization of DNA in quantum terms is to visualize DNA as a simplified theoretical model where each nucleotide is envisioned as a central positive nucleus surrounded by mobile electron clouds. The sugar-phosphate backbone, while chemically monotonous, actually has rather complex electronic structure and the effect of the cloud of delocalized electrons complicates its theoretical description. The extent of delocalization is affected by the conformation of the backbone, though some part of the electronic density is delocalised over the entire backbone due to hyperconjugation effects. Various quantum chemical approximations have been successful at capturing this effect to a variable extent. By comparison, the structure of the RNA backbone is more susceptible to spontaneous cleavage compared to DNA ${ }^{[11]}$.

A similar basic concept was proposed by Rieper and her team whose work supports the proposition that the DNA molecule is held together at room temperature by the quantum mechanical concept of entanglement ${ }^{[18]}$. They visualize DNA as a harmonic oscillator with a negative cloud of electrons moving back and forth relative to the nucleotide, visualized here as a central positive nucleus. When these nucleotides bond to form a base, the electron clouds surrounding them must oscillate in opposite directions to ensure the stability of DNA molecule. These oscillations, or phonons, which are quantum objects, can exist in a superposition states and become entangled; which is exactly what is believed to happen when the phonons are stacked on top of each other in a double helix. While this was initially onceived as occurring at low temperatures, Rieper and her team were able to show that this entanglement can exist at room temperature and is, very likely what is holding DNA together. They found that the wavelengths of phonons are very similar in size to a DNA helix, allowing standing waves to form and based on their model, the effect on the helix is profound. This cannot occur in a purely classical model as the helix would vibrate so much it would shake itself apart; however with quantum mechanics, although each nucleotide in a base pair is oscillating in opposite directions, the fact that this occurs as a superposition of states, rather than an actual vibration means that the overall movement of the helix is zero ${ }^{[18]}$. Other researchers have also echoed similar postulations that the delocalization of electrons can contribute to quantum entanglement distributed among many components of the DNA molecule; and that this contributes greatly to the stability of DNA meaning that DNA has quantum properties, and it has been stated that genetic specificity information are subject to quantum uncertainty limits. Similar to Rieper's work they postulate that this introduces the possibility of arrangement where product protons participate in coupled quantum oscillations and can thus become entangled which contributes significantly to reducing the effect of decoherence and maintaining the stability of $\mathrm{DNA}^{[63,71]}$.

\section{DNA mutagenesis}

Although DNA is a remarkably stable molecule with an incredible ability to ensure accurate handing down of genetic material, there can be errors that occur in the process of replication; spontaneous mutations can occur which result in the gradual adaptation or evolution of a species. Additionally, some mutations can also result in cancer-causing oncogenes. Factors like UV rays and reactive oxygen species can cause DNA damage ${ }^{[72,73]}$, but DNA can still acquire spontaneous point mutations even in the absence of these external factors ${ }^{[74,75]}$.

The structure of DNA is essentially a double helix ladder made up of two helices of nucleic acids bound together by hydrogen bonds. The backbone of the helix is a repeating chain of phosphate and sugars with the configuration of the double helix being complementary in such a way that A-G and C-T always pair up, and combinations of 3 nucleic acids coding for a particular amino acid in socalled coding sections of DNA ${ }^{[11]}$.

As mentioned earlier, the hydrogen bonds between the bases on the two strands of DNA are what actually give rise to the double helix structure of the molecule. Based on Watson and Crick's model, the protons involved in these hydrogen bonds in order to maintain the structural stability ${ }^{[67]}$. However, more recent views taking into account quantum principles suggest that these hydrogen ions can actually shift their positions transiently, a phenomenon known as tautomerism ${ }^{[76]}$. When the bases are in their rare tautomeric forms, the protons involved in the hydrogen bond with the complementary base can shift from base to the other - for example, the proton of adenine (A) can jump over to guanine (T). This is believed to through quantum tunnelling. This is illustrated in Figure 1. Under normal circumstances, adenine always pairs with thymine and cytosine with guanine; however if DNA replication occurs during one of these instantaneous tautomeric shifts, the protons and the corresponding bases will be in the wrong orientation ${ }^{[8]}$; which can result in incorrect base pairing - $\mathrm{G}$ will end up pairing with $\mathrm{T}$ instead of A and so on. This mechanism is believed to rely on the quantum principles of superposition and tunnelling and represents a possible explanation for spontaneous mutations. 


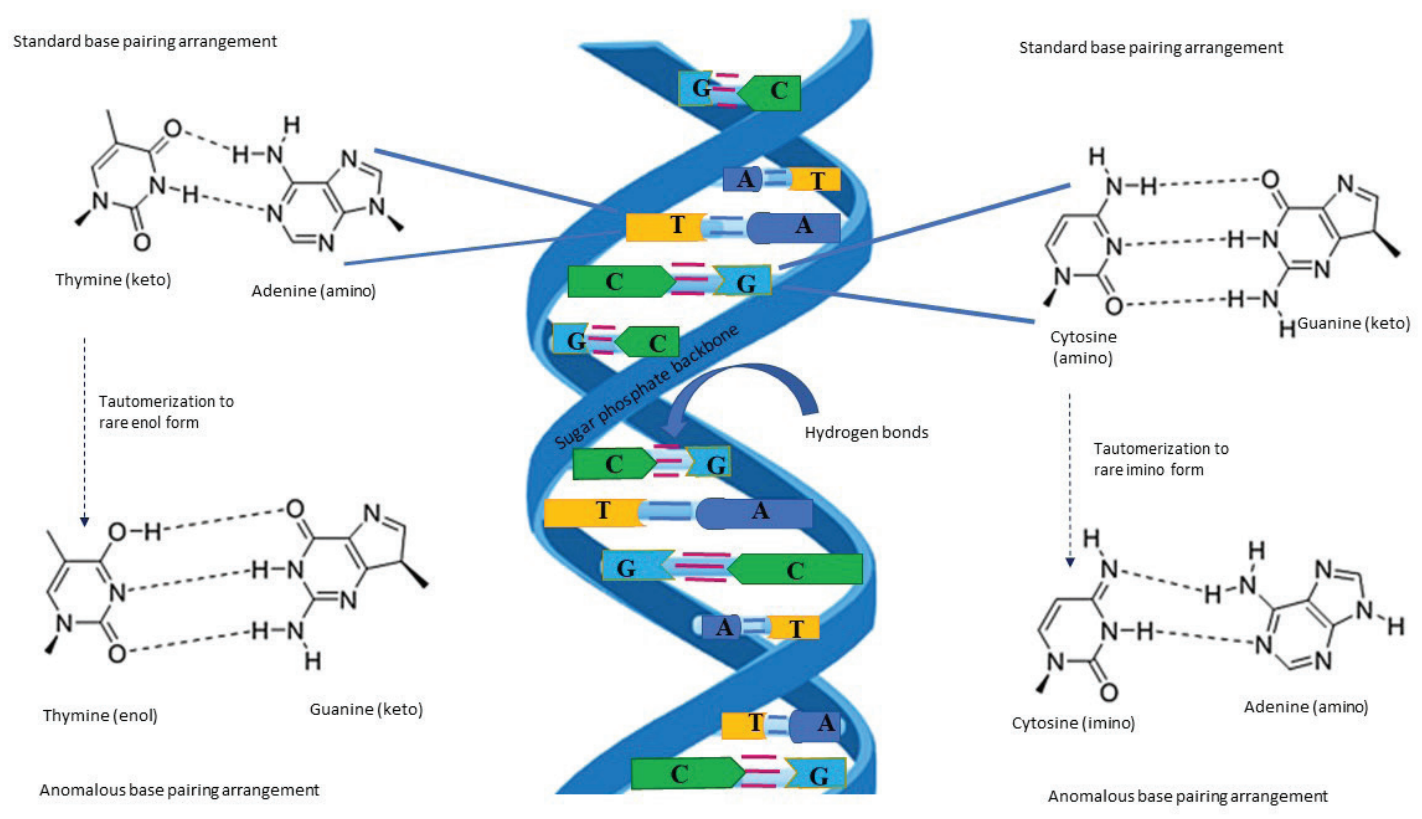

Figure 1. Diagram illustrating double helix structure of DNA molecule with complementary base pairs on the 2 strands, showing the hydrogen bonds between the bases and the changes that take place in tautomerization of the bases which may result in anomalous base pairing which may be the basis of spontaneous mutation.

\section{Adaptive mutation}

Darwinian evolutionary theory is founded on the basis that mutations occur randomly and the evolutionary change occurs based on selection for advantageous mutations conferring a survival advantage. However, this idea has recently been challenged by the finding of the phenomenon termed adaptive or directed mutation. According to McFadden and Al-Khalili, adaptive mutations differ from standard mutations in that "(i) they only occur in cells that are not dividing or dividing only rarely, (ii) they are timedependent not replication-dependent, (iii) they appear only after the cell is exposed to selective pressure" ${ }^{[8]}$. At present we do not have a definitive theory that can satisfactorily account for how a cell can selectively mutate certain genes in response to environmental signals - but the theory of adaptive mutation attempts to explain this.

Adaptive mutation was initially proposed based on work with Escherichia coli bacteria. When a non-lactose fermenting strain was plated on a rich, lactose- containing media, it was observed that lactose-fermenting mutants arose over a period of several weeks. What was intriguing to the researchers was that if mutations truly occur purely randomly, why mutations that did not confer any selective advantage did not develop during the incubation period. Additional support for adaptive mutation came from the observation in parallel experiments where mutants able to ferment lactose arose at much lower frequencies when the organisms were plated on lactose-free media ${ }^{[77]}$.

McFadden and Al-Khalili suggested that adaptive mutation could have its basis in the ability of the proton in the hydrogen bond between two base pairs to exist in tautomeric forms. They postulate that the coding protons may tunnel into the tautomeric positions and subsequently evolve to achieve superposition of simultaneously being in both regular as well as the rare tautomeric forms.
Should cell replication occur while the proton is in the tautomeric position, this will result in a mutant which would then replicate, forming a bacterial colony of mutants. However, cells that fall back into the regular (or non-mutant state) will have their coding protons again in a position to tunnel into their tautomeric state. Interaction with the environment induces decoherence which may then result in the emergence of mutant states, which is the proposed basis of spontaneous mutation. Adaptive mutation is suggested to occur at a much increased rate due to the presence of the environmental driving force such as placing non lactose fermenting Escherichia coli in lactose containing environment; as these adaptive conditions, will create conditions favouring the mutants to precipitate out of the quantum superposition at an increased rate compared to what would happen under 'normal' or non-adaptive environmental conditions ${ }^{[8]}$.

A slightly different way of expressing this is that entanglement occurs between the movement of the individual protons within the DNA bases and the environment. In essence, the environment performs a quantum measurement of the position of the target proton, thus influencing the dynamics of the quantum system in keeping with Heisenberg's uncertainty principle. Cooper ${ }^{[71,78]}$ showed that evolutionary pressure is better described by the probability laws of quantum physics compared to classical kinetic laws ${ }^{[79]}$.

Adaptive mutation is postulated to have a role in crucial changes in microbes that play a role in a range of significant health issues. A high degree of genetic variation has been found on analysis of populations of $H$. pylori isolates from different geographical origins. Quantum mechanics are believed to play a role in this adaptive mutation based on host characteristics ${ }^{[80]}$. Adaptive mutation also plays a role in the development of antibiotic resistance in bacteria ${ }^{[81-83]}$, which represents a brewing global healthcare crisis with 
no clear solution in sight. Mickiewicz found that bacteria can rapidly transition from the walled state to L-form during challenge with a cell-wall-targeting antibiotic, and can then efficiently transition back to the walled state following antibiotic withdrawal. These findings suggest that L-form switching is a physiologically relevant phenomenon that may contribute to the clinical problems such as recurrent infections in high risk populations $^{[84]}$.

Although there is support for the theory of adaptive mutation, there are also those who question whether or not it is a genuine phenomenon. Svesson and Berger are among the skpetics, having stated that they found "little support for mutation bias as an independent force in adaptive evolution, although it can interact with selection under conditions of small population size and when standing genetic variation is limited, entirely consistent with standard evolutionary theory". They were of the opinion that the observations that led to the theory of adaptive mutation were just a false impression are actually due to natural selection shaping the phenotypic effects of mutations ${ }^{[85]}$. More research will need to be done to determine which view proves to be more accurate, though the truth may well lie somewhere in between.

\section{Cancer and quantum mechanics}

Using the concepts of quantum mechanics to describe oncological processes may provide novel directions in our understanding of cancer. Recently it has been concluded that fields such as oncology, biology and chemistry could greatly benefit from application of principles of quantum physics in order to provide new perspectives and greater insights into cancer. This has the potential to lead to deeper understanding of cancers and the potential for more effective and personalized treatments ${ }^{[86]}$. Applying concepts of quantum mechanics to trying to understand why cells might develop oncogenic mutations, as cells may become cancerous as a way to increase their entropy as proposed by Laster et al who proposed this based on combining the uncertainties of genetic changes as expressed by epigenetic changes and/or somatic mutations with the uncertainties of environmental changes ${ }^{[87]}$.

Researchers have established a quantum dynamic framework to explain the theory of mutation, landscapes of biological energy, patterns of cell communication and the cancer interactome under the influence of quantum chaos. Uthamacumaran was able to establish a multidimensional model of quantum evolution by adaptive mutation in cancer biology using a mathematical approach which aimed to identify key signalling pathways required for the phenotypic reprogramming of cancer through an epigenetic landscape, as well as help to understand the cancer interactome mathematically by linking up experimental and theoretical oncology ${ }^{[88]}$.

The potential applications of the principles of quantum physics in oncology have been demonstrated in several areas. Significant advancements in oncology research have been achieved through modelling of concepts such as quantum metabolism, quantum biology, quantum chemistry, quantum biochemistry, and quantum entropy, which have been used to provide new insights into the origin of cancer cells and their rapid, uncontrolled rates of cell division ${ }^{[86]}$. Quantum theory has been shown to be relevant for developing models with the potential to explain cancer related biological and biochemical processes cancer including the effects of carcinogens on DNA, the mechanism of interactions of chemotherapy drugs with genetic material ${ }^{[89]}$; as well as the understanding of DNA mutations and defective protein synthesis ${ }^{[0]}$. If it is possible to devise a way to mathematically model the behaviour of cancer cells, this could potentially facilitate a deeper understanding of a patient's unique cancer and possibly lead to the development of more targeted and personalised treatment specifically adapted for a particular patient ${ }^{[88]}$. Aside from therapeutic applications, the principles of quantum physics may also be able to aid with diagnosis by improving the efficiency and sensitivity of ability to detect diseased tissue through the development of quantum cascade lasers ${ }^{[91]}$.

Quantum theory may even hold the promise of reversing cancerous changes at the cellular level. Biava and her team made the remarkable finding that they were able to use stem cell differentiation stage factors (SCDSFs) to reprogram cancer cells through influencing the microenvironment of the cancer cells. The model they used was based on quantum field theory (QFT) in which SCDSFs are able to prevent cancer from developing by triggering symmetry breaking processes during the development of cancer. These symmetry breaking processes targeted by the SCDSFs lie at the root of many phenomena in elementary particle physics and condensed matter physics, and in the more macroscopic world view, control cell differentiation by governing the phase transitions of totipotent stem cells to higher degrees of diversity and order. Cancers share many similarities with embryonic cells especially with reference to genomics and metabolic profile; and Biava and her team believe they can stimulate dedifferentiation which signifies the reversion from cancerous form back to health and nonproliferation. The high water content in cells may have a role in this as coherent effects have been shown to exist in biological water, which are derived from a dissipative QFT framework, may be a good target in developing novel targets for diagnosis and treatment at a systemic level ${ }^{[92]}$.

An alternative quantum based approach to cancer therapeutics involves quantum dots - small semiconductor spherical nanoparticles composed of heavy metals or organic materials ${ }^{[93,94]}$. Quantum dots have diameters of a few nanometres in size and demonstrate quantum principles such as quantum confinement, quantum tunnelling and semiconductor mechanics ${ }^{[93]}$. Quantum dot technology is believed to have the potential to enable surgeons to accurately differentiate cancerous cells from health cells which would markedly improve the success rates of complete resection of cancerous tissue during surgery ${ }^{[38]}$. These nanoparticles can be modified to enable them to target malignant tumour cells and cancerassociated microenvironments with high specificity and affinity through conjugation with targeted ligands such as 
monoclonal antibodies, peptides or small molecules ${ }^{[95,96]}$.

The lack of ability to maintain a stable and coherent statea within cells is linked to lack of ability to generate sufficient energy levels to maintain order in the cell, particularly in maintaining the order in our DNA and has been linked to aging and cancer ${ }^{[97]}$. In oncology, the phenomenon of the abscopal response where treatment of one malignant lesion had an effect on other lesions distant from the lesion being treated has been described. This has been proposed to be linked to quantum entanglement though at the moment most scientists believe this is related to immune system reactions rather than quantum phenomena $^{[98-101]}$

\section{Applications of quantum physics related to DNA}

Given that quantum physics seems to have a significant role in maintaining DNA stability to allow for the remarkably stable inheritance of hereditary traits across generations, which is seemingly in violation of the laws of thermodynamics; it seems logical by extrapolation that being able to manipulate or adjust 'settings' of the DNA at a quantum level may allow the prevention of pathological adaptive mutations, or possibly even their reversal from pathological to normal. This would then have enormous and wide-ranging implications from treating cancer, to preventing pathogenic strains of microbes emerging in the microbiome to treating antibiotic resistance. There is, in fact, a growing body of literature showing that quantum dots have potential to be used as treatment for multidrug resistant bacteria ${ }^{[102-104]}$ as well as being their potential as a treatment for cancer as mentioned above.

\section{Tunnelling in biological systems - redox reac- tions and enzyme catalysis}

Enzymes are biological catalysts, without which life could not support itself. Enzymes have evolved to allow reactions that require high energy to occur at ambient conditions with a specificity rarely matched by other catalysts ${ }^{[64]}$. The source of all cellular energy, and therefore the basis of life as we know it, originates from reduction-oxidation (redox) reactions, which are central to basic functions of life including metabolism and respiration; and enzymes have key roles in linking up the series of redox reactions in cells. A redox reaction involves the transfer of electrons between two chemical species where is simultaneous oxidation (loss of electron) and reduction (gain of electrons). Recent work in redox biology shows that redox elements are present throughout metabolic and structural systems and they support the genome as it adapts to cope with environmental resources and challenges during the organisms lifespan ${ }^{[105]}$. Given their key role in maintaining the processes central to life, it is perhaps not surprising that it now proposed that alterations in redox balance contribute to aging and disease progression ${ }^{[106]}$.

Energy generation is key to the maintenance of life. From a thermodynamic viewpoint, a cell can be thought of as a semi-open system that allows energy to enter and waste to leave. The energy is required for the cell to maintain order If a cells energy levels fall, this will disrupt cellular order and function, ultimately leading to cellular death and death of the organism. Redox reactions lie at the heart of energy generating metabolic processes in the body, while enzymes catalyse many key reactions to enable survival which could not normally occur under the ambient conditions in the body ${ }^{[43]}$.

The key site where redox reactions take place in cells are the mitochondria, which are, in effect, the power stations of the eukaryotic cell. Mitochondria are the site of oxidative phosphorylation, the process by which ATP, or the basic unit of cellular energy is produced ${ }^{[107]}$. In mitochondrial electron transport chain, electrons are passed along a series of electron acceptors/donors (oxidants and reductants) from $\mathrm{NADH}$ to $\mathrm{O}_{2}$. Collectively, these redox reactions have a wide range of key roles in cellsdriving molecular pumps, maintaining concentration gradients across membranes, and generating energyrich metabolites. Unsurprisingly, oxidoreductases form a significant group of well-represented enzymes ${ }^{[108]}$; and given their central role in processes key to life, disruption in their function is likely to result in disease.

Oxidative phosphorylation is made up of two interrelated components: the electron transport chain and chemiosmosis. The electron transport chain consists of a series of proteins and organic molecules located in the inner membrane of the mitochondria. Electrons are passed along the various components of the transport chain in a series of redox reactions ${ }^{[57]}$. The energy released by these reactions is captured as a proton gradient, which is then used to make ATP. This is called chemiosmosis, a term which can refer to any process where energy is stored in a proton gradient is then used to do work. Chemiosmosis actually accounts for over $80 \%$ of ATP made during glucose breakdown in cellular respiration ${ }^{[109]}$. However, this process is not unique to cellular respiration and is also integral to the light reactions of photosynthesis.

Proton transfer is a key feature of several enzyme-catalysed reactions, where protons move from one molecule to another; with this process believed to be occurring through the quantum-mechanical phenomenon of tunnelling, a phenomenon whereby a particle passes through an energy barrier rather than having to gather the energy to 'climb' over it as illustrated in Figure 2. Proton (effectively hydrogen) tunnelling is known to have an important role in a broad range of biological enzymatic catalytic reactions ${ }^{[7]}$. An example of the molecules involved in this are the three proton-translocating complexes -complex I (CI, NADH:ubiquinone oxidoreductase), complex III (CIII, ubiquinol-cytochrome $c$ oxidoreductase), and complex IV (CIV, cytochrome $c$ oxidase) - which are part of the mitochondrial complex, and are connected by the mobile electron carriers ubiquinone $(\mathrm{Q}$, ubiquinone-10 in humans) and cytochrome $c$ (cyt $c$ ), which then catalyze electron transfer from $\mathrm{NADH}$ to $\mathrm{O}_{2}$. There are several other pathways that also contain enzymes that reduce ubiquinone to ubiquinol and therefore function to feed electrons into the respiratory chain. The proton translocation by CI, CIII, and CIV generates an energy gradient across the inner membrane that is used by enzymes to synthesize $\mathrm{ATP}^{[110]}$. 


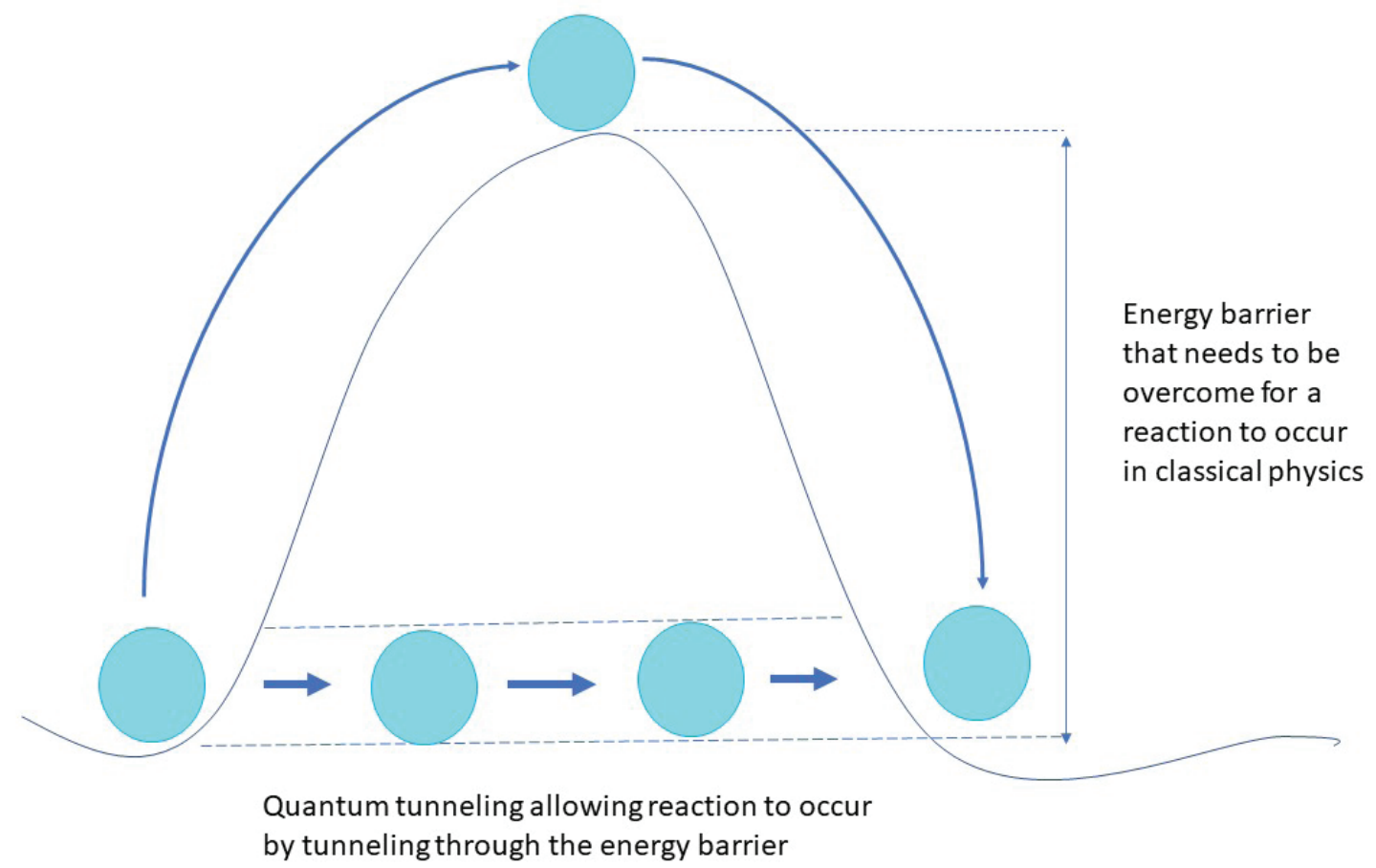

Figure 2. Diagram illustrating the principle of quantum tunneling which is believed to the basis by which catalysts allow reactions that would not normally be able to occur under ambient conditions.

The process of proton-coupled electron transfer is very common in biology and is key to making difficult chemical transformation possible. Given that there is evidence that these occur through long range electron tunnelling and hydrogen tunnelling respectively, this process hinges on quantum mechanics, and this is process can be considered an opportunity for coherence in the process of coordination of the electron and proton transfer ${ }^{[40]}$. In fact, virtually all important biological processes involve quantum biological electron transfer (ET); including DNA repair, cellular respiration, photosynthesis, cellular homeostasis and cell death ${ }^{[111]}$. The fact that this is so truly remarkable because these electron tunnelling across the distances that occur in biological systems would be impossible in a vacuum ${ }^{[2]}$.

In essence, the current understanding we have of quantum systems indicates that in the context of catalysis, the interaction between substrate and catalyst can be considered to be a quantum measurement problem. When the degree of entanglement experienced by catalytic and non-catalytic outcomes of superimposed states are considered, it seems reasonable to postulate that quantum contributions are likely to have played a key role in allowing the efficient operation of catalysts that would then become the basis for the emergence of life $^{[110]}$.

\section{The mitochondria in human disease}

In eukaryotic organisms, including humans, the mitochondria represent the powerhouses of the cell and the site of the electron transport chain based redox reactions that power the cell. Many human diseases have their root cause in disorders related to the mito- chondrial respiratory chain and the enzymes regulating these processes. The respiratory chain involves enzymes that catalyse the various steps in the process of oxidative phosphorylation. Given the central role that oxidative phosphorylation plays in cellular energy metabolism, it is only logical that deficiencies in the enzymes that catalyze this process are important contributors to pathology in humans, having a role in rare inherited conditions as well as common age-associated diseases ${ }^{[112]}$. These enzymes in turn utilize the concept of quantum tunnelling to function; and these enzymes are made based on the blueprint in our DNA which as we saw utilizes many elements of quantum mechanics to preserve its stability.

The DNA encoding for the proteins of the respiratory chain comes from both the mitochondrial DNA as well as nuclear $\mathrm{DNA}^{[113,114]}$. Over 100 mutations of mitochondrial DNA (mtDNA) have been linked to human disease. Mammalian mtDNA encodes for 13 proteins, which are all components of the respiratory chain, which, as alluded to earlier, is crucial for regulated cellular functioning ${ }^{[115]}$. The spectrum diseases that can arise from mtDNA mutations is extremely broad, from with isolated deafness, diabetes and ophthalmoplegia, to complex encephalomyopathic disorders that may include dementia, seizures, ataxia and stroke-like episodes. Diseases of infantile onset with poor outcomes of early death typically involve mutations of nuclear genes encoding subunits of the respiratory chain $^{[116]}$. Given that the concepts of tunnelling, coherence and entanglement do have links to DNA and DNA is the blueprint for the proteins that make up the respiratory chain, it follows that are the ideas of quantum physics as they apply to DNA may also have links to diseases of the respiratory chain. 
As mentioned earlier, the respiratory chain essentially consists of a series of molecules which function as an electron transport chain, where there is combustion of substrate by oxygen. The biological system then extracts energy(via coupled photon transfer) from this electron-transport which is then used to phosphorylate adenosine diphosphate (ADP) to adenosine triphosphate (ATP), as well as to move ions across membranes ${ }^{[43,56]}$. Photosynthesis also utilizes similar principles of electron transfer chains; and of course, the process of movement of an electron, a subatomic particle, which clearly demonstrates quantum behaviour is likely to be able to utilize quantum principles - provided coherence can be maintained within the cell. Recently, it has been found that electromagnetic fields generated by mitochondria are capable of generating 'water order' which is able to protect against decoherence; this could be viewed as evidence of how life has evolved to be able to utilize quantum principles under conditions that seem unlikely based on our current understanding ${ }^{[17]}$. This tenet of quantum biology appears to be gaining support from a variety of sources including from emerging mathematical models that suggest quantum coherence can be maintained for significant periods of time; that coherence can in fact be maintained for orders of magnitude longer in complex biological systems than in simple quantum systems at room temperature ${ }^{[118]}$.

Additionally, an important source of an enzymes catalytic power is the specific nature of the electric field generated by a protease at its active site; and it appears that for calculations to determine this electric field, a quantum approach is much more predictive than a classical approach. Results using quantum-based calculation methods to predict mutation-induced electric field change was much closer to the experimentally measured values compared to the results obtained from conventional force field based equations. The authors suggest that this indicated that quantum effects such as polarization play an important role in accurately describing the electric field inside proteins ${ }^{[119]}$. This suggests that quantum mechanics is key in understanding enzymes, and thus is likely to have a key role in respiration in cells as well as other processes essential to homeostasis.

Given that many human diseases have their roots in damage to the respiratory chain, understanding how these enzymes function is crucial before we can then understand how they may malfunction, and if quantum physics is truly key to their functioning, this may open new realms in potential treatments; as well as to discover potential features that could be used for the construction of novel synthetic oxidoreductase molecules with desirable properties.

\section{Olfaction}

Our sense of smell is another biological function that might owe its function to quantum physics. The traditional model of olfaction involves physical binding of molecules of a specific shape binding to their respective molecules thus triggering neural signals that are detected as specific odours. However, a new and controversial theory of olfaction involves quantum phenomena and suggests that smell originates from the sensing of molecular vibrations by structures in the nasal mucosa; this process is proposed to involve electron tunnelling between the odorant and the receptor to which it binds in the nose ${ }^{[120]}$. Turin proposed that the inelastic tunnelling of electrons from donor to acceptor molecules is proton assisted ${ }^{[121]}$. Recently, a model proposed by Brookes presented evidence that this proposed proton assisted electron tunnelling mechanism fits the observed features of smell. However, it although the theory involves some features of quantum theory, it is worth noting that it is in some ways semi-classical, as it does not depend directly on quantum coherence or superposition $^{[5]}$.

Experimental support for the quantum theory of olfaction comes from work suggesting that our sense of smell seems to be more dependent on the energy spectra of key molecules in order to distinguish scents rather than their shape. For example, a molecule that does not actually contain sulphur but possesses a vibrational spectrum at the same frequency as a sulphur-hydrogen stretch mode was perceived to smell of sulphur ${ }^{[36]}$. This finding is at odds with the traditional lock and key model and it would seem that quantum mechanics is the only mechanism that can explain this vibrational sensitivity of the sense of smell. So far, the strongest available evidence that this mechanism is true is the ability of fruit flies (drosophila) to detect the difference between an organic molecule and a deuterated version of the same molecule; if smell were truly based only on shape recognition, hydrogen and deuterium should be indistinguishable ${ }^{[122]}$.

\section{Clinical implications of olfaction}

Anosmia or hyposmia (complete or partial loss of sensation of smell) is seldom given emphasis as a medical condition, but loss of smell is a common problem in the population and can have significant impact on mental health and quality of life. Loss of sense of smell has been shown to be associated with significant reduction in quality of life and impairments in food intake, safety, personal hygiene, and in their sexual life ultimately leading to depression. Research focusing on olfactory loss suggests that about $20-24 \%$ of the population experience this problem, with a slight increase with advancing age ${ }^{[123-125]}$. Given that this problem affects almost a quarter of the population makes it a clinically significant issue, especially as it linked to deterioration in quality of life and mental health. There are many causes for loss of smell with the most common ones being post viral upper respiratory infection and nasal/sinus disease, followed by head trauma, exposure to toxins/drug, and congenital anosmia ${ }^{[126,127]}$. It is also a component of a variety of illnesses such as Parkinson's disease and recently has been documented as an early sign of COVID 19 infection ${ }^{[128]}$.

Loss of appetite and decreased food appreciation among the elderly is strongly associated with loss of olfactory sense, and is linked to poor nutritional status, loss of weight and increased risk of chronic disease ${ }^{[129-134]}$. Given the significant mental and physical consequences of anosmia and hyposmia, exploring a new understanding of this often-underappreciated sense may yield tremendous gain in prevention, diagnosis and treatment of deficiencies 
in sense of smell.

\section{Nerve transmission and integration of infor- mation in the brain}

The nervous system represents a key area of interest to clinicians and medical researchers, and much about its function is still not fully understood. Many elements of neuronal function whether as individual entities or as collective processing units in the brain have been suggested to require quantum mechanics. The action of neurotransmitters has been suggested to involve tunnelling similar to the mechanism proposed in olfaction. There is also a recent theory suggesting that quantum entanglement between nuclei of phosphorus molecules in vitro might influence neuronal firing. These are among the theories that have contributed to a growing field of study investigating whether quantum effects might truly have a significant contribution to neural processing ${ }^{[55]}$.

Neurons are the basic functional unit of the peripheral as well as central nervous systems, and they are responsible for transmission of information to and from the brain. Exactly how the brain processes and integrates all the peripheral information brought in from the various sensory receptors and combines that with learned information, memories and experiences is still very poorly understood, but there are several proponents supporting the idea that quantum mechanics may have a significant role in this, as well as in the more nebulous concept of consciousness. However, the quantum mechanics, while perhaps most evident and clearly demonstrable in the brain are in many ways applicable throughout the body as these quantum processes take place at all neurons and synapses.

Neurons are the functional unit of both the central and peripheral nervous systems. Given that the means of transmission of information along the axons of nerves and from one neuron to another depends on the movement of ions across the cell membrane, this seems to be very much a quantum mechanical process. This may occur via sodium and potassium channels either as an action potential transmitting information along a single neuron or by triggering release of neurotransmitter for communication across a synaptic gap with another excitable cell. This could be a simple conduit of information or become a part of a complex interactive network determining outcomes very different from the original inputs.

\section{Neuronal communication and quantum me- chanics}

An action potential is generated when the neuronal resting membrane potential is depolarized to threshold which results in mass opening of voltage gated sodium channels which then propagates along the entire axon. The resting membrane potential is maintained by sodium and potassium channels. When an action potential reaches a synapse, it triggers the release of neurotransmitter by triggering voltage gated calcium channels to open. These neurotransmitters, depending on whether they are excitatory or inhibitory may either trigger ion movement in the efferent neuron to be hyper or depolarized. Thus, it can be surmised the basis of neuronal communication and information transmission throughout the body depends on the movement of ions, tiny charged particles, through specific channels - a mechanism which is highly likely to involve quantum mechanics for a complete explanation of its function. The involvement of quantum mechanics may also offer an explanation for how depolarization can potentially spread over a greater area. This phenomenon is greatly limited by myelination of the axons as this reduces tunnelling of potassium ions- this may be one reason for seizures may occur in patients with demyelinating disease ${ }^{[135]}$.

Based on classical theory, the ions responsible for neuronal function pass through their respective ion channel in such a way that each ion passes through one ion channel. However, from a quantum perspective, one ion has an effect on other ion canals in neighbouring ion channels. Given that on average, an ion canal is approximately 1.2 $\mathrm{nm}$ in size with the interior of the canal being about $0.3 \mathrm{~nm}$, and using the quantum uncertainty principle to calculate that a calcium ion has an uncertainty of $0.04 \mathrm{~cm}$, this area would encompass a large number of ion canals; and it is noteworthy that the area of uncertainty of a calcium ion is effectively 100 million times the diameter of the ion itself. Given that there are billions of calcium channels in the brain, the uncertainty arising from this would be truly incredible. Of course, a similar argument would apply to other ions such as potassium which has a De-Broglie wavelength of $0.05 \mathrm{~nm}$ and it has been calculated that it can perform quantum tunnelling and diffraction through an ion canal $0.25 \mathrm{~nm}$ wide. This would then infer that all ions are linked; and this argument can be expanded further to include neurotransmitters whereby a single neurotransmitter molecule, has effects not only on the receptor it is physically bound to, but in fact also affects the other nearby receptors. This can be compared to a key going into a lock but not only opening that door, but also having a part in opening other doors. This whole argument points towards the conclusion that neurotransmitters and ions in the neurons and synapses are probably under the influence of quantum entanglement ${ }^{[54]}$; thus quantum physics are likely to be at the heart of all neural activity in our body.

The Hodgkin-Huxley equation is classically what has been used to explain and predict the movement of ions across a cell membrane, however, especially since the advent of more sensitive equipment, it appears that quantum mechanics may be better able to explain the observations made during an action potential ${ }^{[136-138]}$.

\section{The brain as a quantum computer}

The brain is not simply a classical computer system, instead, as Roger Penrose has proposed, it may be utilizing quantum principles to enable it to carry out its complex functions of processing information and generating awareness ${ }^{[139]}$. These quantum theories of the mind have led to a whole new field of science dubbed 'quantum neurophysics ${ }^{\text {'[33] }}$, which is anchored in the idea that our very existence is rooted in the quantum world. The brain 
remains a mystery in many ways, although we have gained significant understanding of the various regions of the brain and their respective functions, how the brain actually makes sense of information, how information is integrated among various sections of the brain, in particular as relates to memory and higher order thinking, and of course how we actually process and maintain consciousness are still largely an enigma. There have been attempts to try to decipher how the vast neural network - with all its connections and chemical communication - can actually give rise to consciousness and cognition which are what we identify as self ${ }^{[5]}$. Given that classical theory cannot make sense of this, it seems likely that the brain is using quantum effects - although the precise mechanism, location and degree is still unknown ${ }^{[43]}$.

Khrennikov et al. used quantum uncertainty principles as the basis for creation of a model of generation of action potentials in neurons; with the uncertainty being a consequence of the complex electrochemical processes in the brain alluded to earlier, particularly with reference to the initiation of an action potential. They postulate that processing of information in the brain depends on a superposition state of neurons being quiescent or firing as opposed to the classical model where a neuron has an all or none behaviour in terms of firing an action potential. Their theory also suggest that a group of neurons performing a particular function is, in fact, an open quantum system which is interacting with the surrounding electrochemical environment ${ }^{[140]}$.

\section{Neurobiology}

The very newly emerging field of quantum refers to a specific branch of quantum biology which is focused on quantum physics in the nervous system in particular with reference to how it may explain the emergence of higher cognitive functions like consciousness, memory, internal experiences, and the processes of choice and decisionmaking. Given that the principles of quantum mechanics like in the nano world, this theory works on the premise that the information processing units of the central nervous system are the smaller quantum processing occurring inside the neurons and forming connections between them; this is contrast to the classical view where the neurons as a whole form the information processing units $^{[33]}$.

These higher elements of consciousness and cognition are largely what make us human and these are elements which are often lost with brain damage from a variety of causes - from brain trauma in a car accident to formation of neurofibrillary tangles of Alzheimer's disease. The notion that quantum physics has a significant role in the highly adaptable, cognitive functions in the brain seems hard to imagine given that the brain operates in conditions far from a stable thermodynamic equilibrium ${ }^{[141]}$; however in spite of these conditions that seem to be a recipe for decoherence, the brain consistently produces outcomes that defy classical mechanics.

Penrose, in particular, has argued that human understanding must involve some elements of quantum computation. Penrose and Hameroff actually proposed a specific model called orchestrated objective reduction which suggested that quantum computation occurs in microtubule protein assemblies in the neurons of the brain, which then results in consciousness ${ }^{[141]}$. Microtubules are basically hollow cylinders made of the protein tubulin which comprise the internal cytoskeleton of cells including neurons ${ }^{[142]}$.

Helmut Schmidt, who spent much of his career studying the effect of consciousness and the observer on quantum mechanical probability, reached the conclusion that consciousness was actually able to interact with random physical processes under certain conditions ${ }^{[143]}$. Although there were few of his contemporaries who were of a similar mind, there has been fairly recent work in other laboratories obtaining similar results ${ }^{[144,145]}$. However, many quantum physicists are hesitant to lend credence to the experimental findings as they are at odds with the existing paradigms.

Perhaps part of the difficulty getting buy in to these theories that consciousness arises as the outcome of quantum interactions is related to the fact that consciousness seems to arise only from large scale connectivity patterns between neurons and only in certain parts of the brain such the cortico-thalamic complex whereas all neurons actually function based on quantum-level events. However, the history of science shows us that revolutionary ideas may not necessarily be wrong in spite of the lack of acceptance by the scientific community. Tarlaci and Pregnolato stated that "over the past fifty years, approximately 800 studies have been published by 68 different laboratories on the effect of consciousness, intention and observation on random processes", and for a significant proportion of these studies, the effect of the observer was significantly reflected in the data obtained ${ }^{[33]}$ which agrees with the basic principles of quantum physics. Although quantum theory seems to be a better fit than classical to explain what we do know about consciousness and cognition, there are gaps in the theory that are unable to satisfactorily account for some observations; suggesting that existing theories are still incomplete. Hopefully as we gain deeper understanding of quantum physics and neurobiology we may unravel the scientific basis for these elusive phenomena; which may then offer new avenues to seek cures for diseases affecting these such as coma and dementia.

\section{Medical Applications of quantum neurophysi- ology and neurobiology}

The understanding of quantum mechanics in the neural networks in the body may have many implications for diagnosis and treatment of a wide variety of degenerative neuronal disorders, genetic defects, demyelinating disease, epilepsy and other seizure disorders, possibly even memory loss and dementia; or even psychiatric illnesses. The application of this has practical examples such as general anaesthesia's ability to switch off consciousness has been suggested to be the result of measured changes in electron $\operatorname{spin}^{[55]}$.

Capturing clear images of the soft tissue of the brain can be a significant challenge. Shi et al were able to demonstrate the preservation of entanglement as one 
photon of a pair passed through different types of tissue slices from rat: brain cortex, brain stem. This may be the basis for quantum based medical imaging, particularly as photon entanglement, provides a level of coherence that is not present in classical correlations ${ }^{[146]}$. This might greatly enhance imaging capabilities, allowing for improved visualization of intracranial lesions.

\section{Photoreceptors}

The ability to detect and respond to a variety of light based environmental sensory inputs through lightsensing proteins plays a vital role in the survival of many organisms. In human beings, the eyes represent the organ responsible for vision with the light sensing proteins in question being the photoreceptors in our retina. All biological photoreceptors contain substances called chromophores which are able to undergo chemical changes upon absorbing photons; transforming from resting to excited states and eventually leading to light-induced signal transduction to the brain via the visual pathway. Given that the entire process hinges on excited-state molecular dynamic, which in turn very much resides in the domain of quantum realm, it then follows that quantum mechanics would be needed to develop models of the dynamics of photoactivation in the retina.

The classic, most frequently studied photoreceptor in the retina is rhodopsin, which contains retinal, a chromophore that undergoes cis-trans photoisomerization following light absorption, which has been described as a 'vibrationally coherent process $^{[147]}$. This process then results in a series of biochemical processes within the photoreceptor that result in a visual signal being generated ${ }^{[148,149]}$. Additional features of this photoisomerization being quantum in nature include the remarkable speed of the reaction $(<200 \mathrm{fs})$ and its high specificity (quantum yield of $\sim 0.65$ ), which are thought to be the result of ultrafast coherent wave-packet dynamics which in turn depend on the symmetries of the electronic states and the quantum mechanical positioning of the excited-state potential energy surfaces ${ }^{[2]}$.

Although it seems only logical that quantum theory should underlie the process of photoisomerization in natural photoreceptors there is limited definitive evidence of this. There has been experimental work modelling the isomerization of retinal using quantum based vibrionically coupled electronic two-state system (effectively a quantum coherent system) which was able to match the known spectroscopic pattern of rhodopsin; this finding also provides a possible explanation for the incredibly high reaction speed ${ }^{[13,150]}$. Some of the evidence available comes from work with bacteriorhodopsin, a protein which is related to the rhodopsin in vertebrates, which was able to demonstrate coherent optical control of retinal isomerization using lasers ${ }^{[151,152]}$. The dynamics of how natural photoreceptors are able to achieve their remarkable efficiency through coherent interference of wave packets of electromagnetic radiation are intriguing as a deeper understanding could be applied to influence chemical dynamics to obtain increased reaction yields.
Beyond the retina, the neural signals that need to be transported to the visual cortex for conscious perception are also likely to be utilizing quantum principles as discussed in the earlier section on quantum applications in nerves.

\section{Possible clinical applications quantum theory of photoreceptors}

Given how crucial vision is and how debilitating even mild loss of vision can be to being able to function effectively independently, gaining a better understanding of the quantum mechanics involved in photoreception may yield new insights into modulating and treating visual dysfunctions; possibly even being able to generate artificial photoreceptors to replace those damaged in conditions such as diabetic or hypertensive retinopathy.

\section{Photosynthesis}

Plants, algae or other organisms such as some bacteria which are capable of photosynthesis form the basis of every food chain due to their ability to make sugars from water and carbon dioxide in the presence of sunlight. These photosynthetic cells, therefore, possess the ability to capture and harness photons, in itself a tantalizing link to quantum theory, and they are able to do it with a remarkable degree of efficiency making them a focus of research for quantum biologists. However, there was always a healthy amount of scepticism as although quantum coherence and resonance was a plausible explanation, the warm, wet, and disordered environment that is the typical state biological systems where the pigment complexes are located was thought unlikely to be able to mirror the highly ordered state of solid-state systems which are able to facilitate delocalization of excitations over an entire crystal ${ }^{[16]}$.

The current understanding of photosynthesis based on several robust experiments strongly supports that quantum physics are operational in allowing the process to occur at its known levels of efficiency. Photosynthesis essentially involves capturing the energy of the photons from sunlight through a light-harvesting mechanism, in this case, chlorosome antenna. Once captured by this antenna, the absorbed excitation energy from the sunlight is transferred to a photosynthetic reaction center via the FennaMatthews- Olsen (FMO) complex - a pigment protein complex - which links the antenna and the reaction center ${ }^{[153]}$. The FMO protein complex is made up of seven chlorophyll molecules located in a protein cavity ${ }^{[154]}$. These chlorophyll molecules are able to transiently store the energy captured by the antenna as electronic excited states in order to channel it to the reaction center, which can be thought as a solar cell. The ability of the FMO complex to 'wire'this energy between the light harvesting antenna and the reaction center has been studied in green sulphur bacteria which are photosynthetic organisms where it has been found that the energy absorbed is passed over the seven bacteriochlorophyll molecules in each FMO complex prior to reaching the reaction center ${ }^{[155]}$. Recently, researchers have suggested that this energy transfer relay is facilitated by quantum mechanics ${ }^{[40,47,107]}$. An overview of the process is illustrated in Figure 3. 


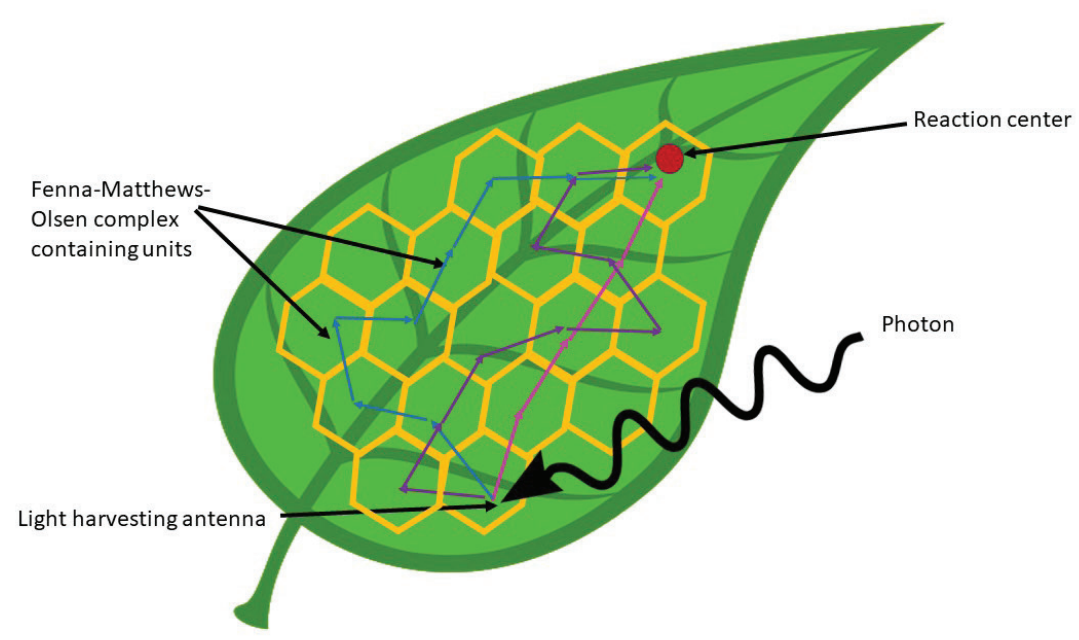

Figure 3. Diagram illustrates the theory of how photons are capture by light harvesting antenna, and sent to the reaction center. The multiple pathways indicate some of the possible pathways the light could take which all exist simultaneously due to superposition.

Scientists used to visualize the photon of light captured would hop from bacteriochlorophyll molecule to molecule along a single pathway down an energy gradient; but it was visualized that the energy transport would occur in a random process, jumping from one FMO complex to another incoherently, as Lambert so descriptively likened to "a drunken sailor staggering downhill". However, by calculation, this would yield only about $50 \%$ efficiency, and cannot account for the close to $100 \%$ efficiency of light transfer of photons from antenna to reaction center. Given that random motion was unsatisfactory to account for the observation of the almost-perfect efficacy of light transfer, biophysicists postulated that, the energy most likely exists in a quantum superposition state. This would enable the energy to simultaneously travel along all possible pathways (see Figure 3) - in much the same way a quantum computer has the ability to access all the data in a particular database at the same time. The implication here is that the wavefunction of the exciton is in a state of coherent superposition over the molecules, which facilitates optimization of the time taken for the absorbed light energy to travel towards the reaction centre. Based on this theory, once the shortest, most direct road is identified, the system "snaps out of superposition" and utilizes the route identified, making it possible for the energy to take the most efficient path every time ${ }^{[2,156]}$.

Engel and his team were the first to show pigment protein from photosynthetic organisms was able to demonstrate long lasting coherence at physiological temperatures ${ }^{[12]}$. Their experimental work was able to capture the spectroscopic observation of quantum coherent dynamics of transfer of an exciton being transported across the multiple pigment molecules within the FMP complex, although this was at low temperature $(77 \mathrm{~K})$. Since this landmark work, a large number of papers have been published on this topic, with experimental work even suggesting that the coherence found is non-negligible for up to $300 \mathrm{fs}$, even at room temperature ${ }^{[2]}$. For example the FMO protein-chlorophyll complex of green sulfur bacteria demonstrates coherence for times in excess of $1000 \mathrm{fs}$ even at high temperature $(277 \mathrm{~K})$ in the $2 \mathrm{D}$ Fourier transform electronic spectroscopy ${ }^{[40,154]}$. The earliest measurement of quantum coherence in photosynthetic organisms was carried out at temperatures far below room temperature ${ }^{[12]}$. However, it has been demonstrated that quantum beating can be demonstrated at room temperature after exciting the photosynthetic complexes with a laser pulse ${ }^{[6,154,157,158,159,160]}$.

Overall, it can be said that work on photosynthetic organism has provided substantial evidence of quantum effects at room temperature, something that was considered virtually unthinkable not so long ago. There is considerable evidence the coherence demonstrated in these biological systems is capable of enhancing the efficiency of photosynthetic units, such as the FMO complex. This enhancement may only be by a few per cent, but this may confer significant survival advantage to certain species which live in low-light conditions, such as green sulphur bacteria or algae. There is still debate as to whether quantum mechanics truly evolved purposefully to enhance generation of energy is not yet clear but the fact that this ability is found across a range of organisms suggests it evolved with a purpose.

\section{Applications of photosynthesis and quantum biology}

A better understanding of the mechanisms plants and other photosynthetic organisms have developed to facilitate ultrafast energy transfer and to maintain this in the desired direction with high quantum efficiency might hold important clues about how to develop systems with the capacity to effectively generate electricity through conversion of solar energy to electrochemical energy. From a clinical standpoint, many medical technologies require high electrical energy to drive them and finding effective ways of generating energy would help tremendously, especially in bringing these medical technologies to remote areas lacking electricity, if solar energy could be harnessed more effectively. 


\section{Avian magnetoreception}

Magnetoreception refers to the ability that that some migrating species - such as migratory birds- possess to use the Earth's magnetic field to navigate their flight path $^{[161]}$. It had previously been suggested that this could be related to deposits of magnetic iron minerals - such as magnetite- in these organisms, however recent reports suggest that even if these deposits do exist they do not seem to play in a role in the magnetic sense $\mathrm{e}^{[19]}$.

There has been growing evidence to suggest that the phenomenon of magnetoreception may be related to quantum phenomena ${ }^{[15,62,162]}$. The magnetic sensor in migratory birds has been shown to be activated when light strikes their retina ${ }^{[1]}$; suggesting that this magnetoreception is photoreceptor based, as indeed has been demonstrated in European robins. Specific frequencies in the light entering the eye have been shown to have demonstrable effects on this system; and the magnetoreception system seems to be responsive to alterations in the intensity of the external magnetic field, but the system was able to readjust to these changes over time. Additionally, the ability of these species to navigate was disrupted by magnetic pulses, and also by very weak external oscillating magnetic fields. In support of the fact that the relatively weak magnetic field of the Earth is adequate to create meaningful effects, and that these species could genuinely be using this in the normal environment, Bandyopadhyay et al. were able to demonstrate a reasonable parameter regime where the sensitivity migratory birds ability to decipher direction is enhanced by environmental noise, suggesting that it is not necessary to be able to generate long coherence times for this system to function, and may, in fact, even diminish its efficacy ${ }^{[15]}$.

Researchers' current best guess at explaining the basis of this is the radical-pair mechanism. This refers to a mechanism whereby the energy absorbed from each incoming photon creates a pair of free radicals which are highly reactive molecules, each of which contains an unpaired electron ${ }^{[1,163]}$. These unpaired electrons each possess an intrinsic angular momentum, or spin, the orientation of which can be altered by a magnetic field these spin correlated states are in either singlet or triplet configuration $^{[36]}$. All matter does in effect have matter waves (shown by De Broglie), and the wave function for these waves possesses 'phase', meaning that quantum particles essentially behave as a rotating cloud, and can be influenced by magnetic fields; then these particles have 'spin' ${ }^{[164]}$. As the two components of the radical pair move further apart, each one is primarily influenced by varying magnetic fields - one by the magnetism of the nearby atomic nucleus, and the other which is further from the nucleus, is influenced by the Earth's magnetic field ${ }^{[7]}$. The variation in the field shifts between the components of the radical pair shifts the radical pair between two quantum states each of which has a different chemical reactivity; which then provides a possible mechanism by which the radical-pair functions as a biological compass ${ }^{[165]}$.

At present, the exact nature of the radical pair that could be involved in this mechanism has yet to be determined, however, radical pair reaction is known to occur within the cryptochromes of the eye; and it is thought to be highly probable that this could be represent the means by which incoming light could be the trigger for both vision as well as navigation using the magnetic field ${ }^{[166]}$. Using simple models based on the these sort of radial pairs, based on pairs with highly anisotropic spin configurations, it has been demonstrated in principle that the reaction products of the radical-pair reaction described is sensitive to the direction of an external magnetic field; and is capable of reproducing the disruptive effect of time-dependent external magnetic fields at frequencies in the radiowave portion of the electromagnetic spectrum ${ }^{[163]}$.

This is relevant to quantum biology as the spin singlet and triplet state are some of the most quantum of states; and spin is one of the properties of shared by coherent particles. The single and triplet spin states are equivalent to Bell states, which are states at the maximum possible degreed of entanglement; a property that is considered highly desirable in quantum information systems ${ }^{[2,164]}$.

So far, one caution that been raised regarding this theory is that manmade models have not been able to demonstrate the ability to sustain quantum coherence and entanglement properties for a sufficient length of time that would be required to generate meaningful effects in magnetoreception. However, work by Maeda et al. ${ }^{[167]}$ has been able to demonstrate the most sensitive radicalpair reaction so far with magnetic fields down to stronger than $5 \mathrm{mT}$ are capable of causing conversion between the singlet and triplet states in radical pairs. There has also been research on cryptochrome, the photoactive pigment in the eye that is at present considered to be the most likely candidate for where the radical pair mechanism is located ${ }^{[168]}$. Recently, work done in-vitro on cryptochrome extracted from the plant Arabidopsis thaliana were able to demonstrate magnetically sensitive radical-pair reactions at very weak magnetic fields - $25 \%$ change in yields of order of radicals at $30 \mathrm{mT}$ and $1 \%$ at $1 \mathrm{mT}$. The Earth's magnetic field is typically in the range of $30 \mu \mathrm{T}$ to $60 \mu \mathrm{T}$, which is much weaker than these experimental fields, however it has been suggested that it is possible the response in vivo may be much stronger than what has been demonstrated in the lab ${ }^{[167]}$.

The evidence at this point is still leaves many questions unanswered and may also require work to be done on the neural networks responsible for processing the magnetic field information to help unravel this conundrum.

\section{Future medical applications:}

A deeper understanding of how quantum mechanics plays a role in the various organs of the human body may revolutionize the practice of medicine as we know it. There is a myriad of potential application for quantum theory in clinical medicine ranging from diagnostic modalities to therapeutics including treating cancers, channelopathies, disorders of vision and olfaction, and possibly even novel methods of drug delivery.

At present, though it is not often immediately considered as such, quantum physics is already seen in many aspects 
of medical diagnostics and therapeutics in the form of electromagnetic (EM) radiation. Planck's theorem arose from observations of electromagnetic phenomena and EM radiation is the classic example of wave-particle duality putting it at the heart of quantum theory. EM radiation across the EM spectrum already has many applications in modern clinical practice as summarized in Figure 4 including radiation therapy for cancer ${ }^{[169]}$, imaging as $\mathrm{X}$-rays and CT scans, ultrasound therapy, radiofrequency ablation, infrared ${ }^{[170]}$ and even ultraviolet light for chronic skin lesions unresponsive to standard therapy believed to be related to modulation of the immune system ${ }^{[171]}$. Ultrasound has even been suggested to helpful in assessing lung lesions in patients with COVID-19 infection ${ }^{[172,173]}$. Exploring the various aspects of EM radiations role may be able to extend to prophylaxis as well as therapeutics at appropriate frequencies and doses.

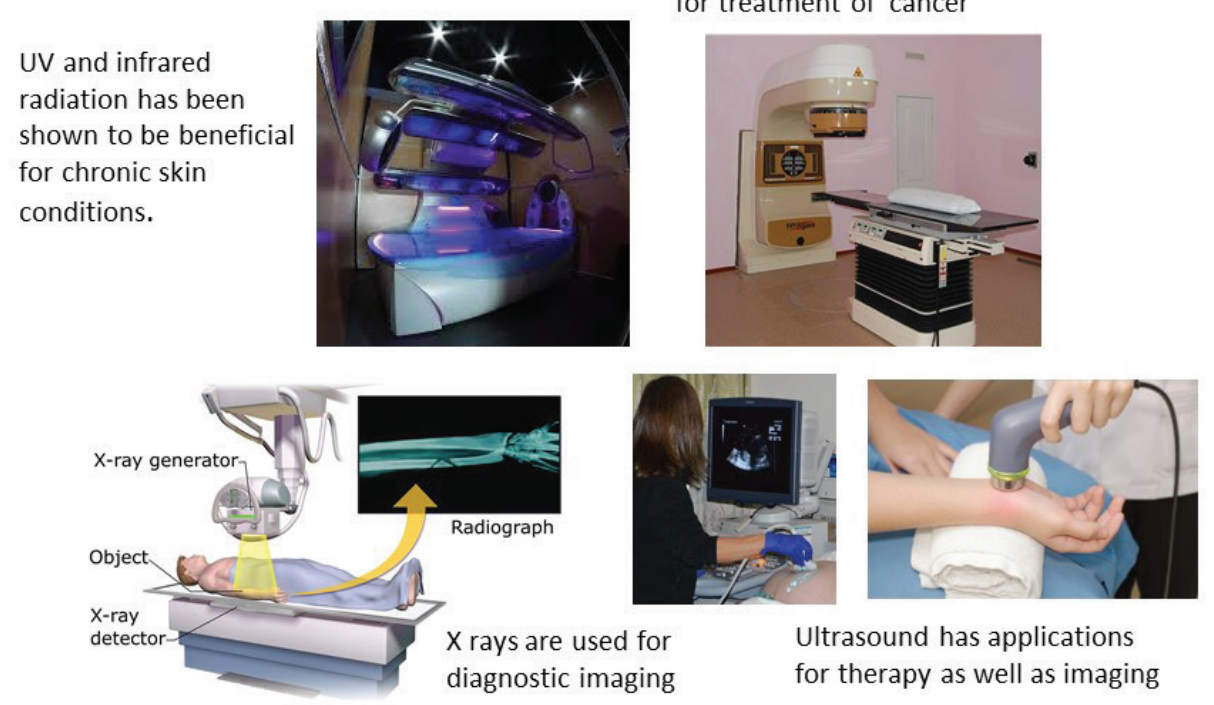

Figure 4. Electromagnetic radiation, in many ways the embodiment of quantum phenomena, are already harnessed for many applications in clinical medicine.

From the material reviewed and discussed, the fact that quantum mechanics is key in maintaining DNA stability may hold a key to finding ways by which we may be able to preserve that stability and prevent oncogenic mutations. If the theory of adaptive mutations involving formation of tautomers is true, it might also be possible to one day exploit that property to convert those tautomers back to their benign form. For example, using quantum field theory model, Biava et al. found they could manipulate cancerous cells to revert to healthy cells with SCDSFs ${ }^{[92]}$. Using quantum dots to treat cancers has already shown promise, in particular in the enhanced ability to differentiate benign cells from cancerous ones ${ }^{[95,96]}$.

Understanding how to manipulate adaptive mutation may also help to curb the formation of antimicrobial resistant microbes and various other pathogenic organisms that arise as a response to their environment. As a direct treatment strategy, light activated quantum dots have shown promising activity against a range of superbugs including Methicillin resistant Staphylococcus aureus, extended spectrum beta-lactamase producing Klebsiella pneumoniae and carbapenem-resistant ${ }^{[97]}$ Escherichia coli $^{[102]}$.

A deeper understanding of drug isomers and how they interact with their target molecules based on combined quantum and classical mechanics may help pave the way to highly potent and effective drugs. One example is in the treatment of Amyotrophic lateral sclerosis (ALS), an irreversible, fatal, progressive neurodegenerative disorder, the root cause of which is believed to be due to a mutant superoxide dismutase 1 (SOD1) gene ${ }^{[174]}$. Resveratrol, a substance which has the capability to inhibit superoxide dismutase (SOD1) and represents a potential treatment ALS, has been shown to have differential levels of interaction with the mutant and wild type SOD1; with resveratrol having higher levels of interaction with the mutant variant. Resveratrol has been shown to be able to stabilize the conformation of mutant SOD2 proteins, which may have the effect of preventing the pathological changes in the nervous system in $\mathrm{ALS}^{[175]}$. There are very small structural differences between mutant and wild type SOD1 proteins ${ }^{[176]}$, and it may be possible that recognition of vibrational spectroscopic patterns may enable the variable interaction observed. If this is true, it may pave the way to seeking a new understanding for more specific therapeutic agents. Quantum mechanics has been used to better understand the stability of the HIV virus ${ }^{[177]}$ as well as various side chain mutations in Burkholderia cepacia, a species of bacteria ${ }^{[178]}$ which may lead to development of more effective therapeutic agents.

Seeking cures to cancer is in many ways a holy grail of modern science and is possible that exploring quantum 
dynamics related to disturbances in oxidative metabolism and coherence may offer exciting new therapeutic options as these issues are central to cancer development. These disturbances lead to disordering of the water molecules in a cell which may then cause disruption of the cell's electromagnetic field ${ }^{[117]}$. Seeking a deeper understanding of this area and aiming to use metabolic-modulating drugs or other modalities such as electromagnetic radiation that could permanently reverse the mitochondrial dysfunction may be of benefit in exploring new treatment modalities that could seek to manipulate cells at a quantum level.

Given the role of the mitochondria and the association between redox disturbances and aging ${ }^{[97,105]}$, and the links with quantum physics in these processes, application of these principles may yield novel therapies in preventing degenerative changes of aging.

Utilizing a deeper understanding of magnetic fields and their quantum effects may also yield more precise methods of imaging to enhance diagnosis. Shi et al found it was possible to preserve the polarization entanglement of the photons fired at the tissue being imaged by spatially filtering the ballistic scattering of an entangled photon. They also found that the degree of entanglement correlated with structure and water content, which may consistent with the idea that cellular water is able to retain coherence ${ }^{[146]}$. This suggests that application of quantum concepts may yield more effective means of medical imaging and diagnosis.

The involvement of quantum tunnelling as the basis of olfaction $^{[5,121]}$ and the quantum mechanics related ability of photoreceptors of the eye to capture even a single photon with remarkable efficiency ${ }^{[13,14]}$, suggests that a deeper understanding of these phenomena may pave the way to novel therapies for conditions affecting vision and smell. The understanding of quantum tunnelling in olfaction may open new avenues to helping improve or regain smell in those with hyposmia; and a deeper understanding of the light harvesting mechanism of the photoreceptors of the eye may yield ways that to aid with regaining vision in those with damaged photoreceptors.

Neurological and psychiatric disorders represent an area of medical science where there has been a struggle to find truly effective therapies. Given the likelihood that quantum physics actually underpins the function of neural activity, neuronal integration and processing of information and even the basis of consciousness and memory ${ }^{[33,54,65]}$ it may be possible to use these principles to explore novel approaches to a wide variety of debilitating conditions. It is conceivable that applications of quantum physics may be able to assist with prevention or possibly reversing memory loss; or to help manipulate consciousness both to induce a medical coma as well as to reverse comatose states. A better understanding of consciousness may also allow induction of anesthesia through methods with less side effects than the present practices. A deeper understanding of quantum mechanics in neural activity may also suggest novel methods of treating conduction disorders of the nerves from demyelinating disease to dysfunctional ion channels to complex seizure disorders.

\section{Conclusions}

Based on the existing literature, it is evident that nature has been able to evolve mechanisms and structures by which to harness quantum mechanics to aid processes necessary to life in ways that were highly unexpected based on the initial findings of quantum physics where these behaviours were thought to only occur under very tightly controlled environmental conditions. From what has been discovered, it seems likely that we have only just begun to unearth the tip of the iceberg in terms of how quantum phenomena play a role in living organisms - perhaps as more sophisticated equipment and testing become available we may be better placed to explore these further.

It seems clear that there are many scenarios where quantum mechanics are at work in the human body, though their exact nature remains to be fully defined. There is also significant debate about whether the phenomena that have been observed truly have a functional purpose thought it seems more and more likely that they do. This then suggests that there are a huge number of potential applications of quantum biology in science and in medicine - in terms of diagnosis as well as treatment of a variety of human maladies.

While there is clearly much more work to be done before we are fully able to understand and apply the principles of quantum physics in a practical way in clinical medicine, the fact that it is clearly involvement in so many key processes for life suggests that this field holds significant clues to unlocking many mysteries of biology. It seems that this joining of forces of physics and biology will produce results neither could produce alone - only time will bring the truth to light and unravel the mysteries of biology leading to improved strategies for healthcare.

\section{Conflict of Interest}

The authors declare that there is no conflict of interest in this work.

\section{Reference}

1. Ball, P., Physics of life: The dawn of quantum biology. Nat, 2011; 474(7351): 272-274.

2. Lambert, N., et al., Quantum biology. Nat Phys, 2013; 9(1): 10-18.

3. Adesso, G., Franco, R.L. and Parigi, V. Foundations of quantum mechanics and their impact on contemporary society. Philos Trans Ser A, Math, Phys, Eng Sci, 2018; 376(2123): 20180112.

4. Zurek, W.H. Quantum theory of the classical: Quantum jumps, Born's Rule and objective classical reality via quantum Darwinism. Philos Trans Ser A, Math, Phys, Eng Sci, 2018; 376(2123): 20180107.

5. Brookes, J.C., Quantum effects in biology: golden rule in enzymes, olfaction, photosynthesis and magnetodetection. Proc Math Phys Eng Sci, 2017; 473(2201): 20160822.

6. Scholes, G. Green quantum computers. Nat Phys, 2010; 6(6): 402403.

7. Marais, A., et al. The future of quantum biology. J R Soc Interface, 2018; 15(148).

8. McFadden, J. and Al-Khalili, J. A quantum mechanical model of adaptive mutation. Biosyst, 1999; 50(3): 203-211.

9. Usselman, R.J., et al. The quantum biology of reactive oxygen species partitioning impacts cellular bioenergetics. Sci Rep, 2016; 6(1): 38543 .

10. Cooper, W.G. Roles of evolution, quantum mechanics and point mutations in origins of cancer. Cancer Biochem Biophys, 1993; 13(3): 
$147-170$.

11. Šponer, J., et al. The DNA and RNA sugar-phosphate backbone emerges as the key player. An overview of quantum-chemical, structural biology and simulation studies. Phys Chem Chem Phys, 2012; 14(44): 15257-15277.

12. Engel, G.S., et al. Evidence for wavelike energy transfer through quantum coherence in photosynthetic systems. Nat, 2007; 446(7137): $782-786$.

13. Sia, P.I., et al. Quantum biology of the retina. Clin Exp Ophthalmol, 2014; 42(6): 582-589.

14. Chakravarthi, R., Rajagopal, A.K. and Usha Devi, A.R. Quantum mechanical basis of vision. 2008; arXiv:0804.0190.

15. Bandyopadhyay, J.N., Paterek, T. and Kaszlikowski, D. Quantum coherence and sensitivity of avian magnetoreception. Phys Rev Lett, 2012; 109: 110502 .

16. Cao, J., et al., Quantum biology revisited. Sci Adv, 2020; 6(14): eaaz4888.

17. Yin, J., et al. Satellite-based entanglement distribution over 1200 kilometers. Sci, 2017; 356(6343): 1140

18. Rieper, E., Anders, J. and Vedral, V. Quantum entanglement between the electron clouds of nucleic acids in DNA. arXiv e-prints, 2010; arXiv: 1006.4053.

19. Ball, P. Schrödinger's cat among biology's pigeons: 75 years of What Is Life? Nat, 2018; 560(7720): 548-550.

20. Wang, C., et al., A Schrödinger cat living in two boxes. Sci, 2016; 352: $1087-1091$.

21. Ranganath, G.S. Black-body radiation. Resonance, 2008; 13(2): 115133.

22. Swanson, M.S., The origins of quantum mechanics. In: A Concise Introduction to Quantum Mechanics. Morgan \& Claypool Publishers; 2018. p. 2-1-2-15.

23. Das, S.N. De Broglie's wave theory and the two-wave description of matter. Il Nuovo Cimento B (1971-1996), 1992; 107(10): 1185-1192.

24. Weinberger, P. Revisiting Louis de Broglie's famous 1924 paper in the Philosophical Magazine. Philos Mag Lett, 2006; 86(7): 405-410.

25. Lima, N.W., et al. Louis de Broglie's wave-particle duality: From textbooks blackboxes to a chain of reference presentation. Rev Bras Ensino Fis, 2020; 42 .

26. Griffiths, D.J. and Schroeter, D.F. Introduction to Quantum Mechanics. Cambridge University Press; 2018.

27. Farrow, T. and Vedral, V. Classification of macroscopic quantum effects. Opt Commun, 2015; 337: 22-26.

28. Roszak, K., Filip, R. and Novotný, T. Decoherence control by quantum decoherence itself. Sci Rep, 2015; 5(1): 9796.

29. Zurek, W.H. Decoherence and the transition from quantum to classical. Phys Today, 1991; 44(10): 36-44.

30. Gerlich, S., et al. Quantum interference of large organic molecules. Nat Commun, 2011;2(1): 263

31. Yu, H., et al. Quantum correlations between light and the kilogrammass mirrors of LIGO. Nat, 2020; 583(7814): 43-47.

32. Zurek, W.H. Decoherence, einselection, and the quantum origins of the classical. Rev Mod Phys, 2003; 75(3): 715-775.

33. Tarlacı, S. and Pregnolato, M. Quantum neurophysics: From nonliving matter to quantum neurobiology and psychopathology. Int J Psychophysiol, 2016; 103: 161-173.

34. Walls, D.F. and Milburn, G.J. Quantum coherence and measurement theory. In: Walls D.F. and Milburn, G.J. (editors). Quantum Optics. Berlin, Heidelberg: Springer Berlin Heidelberg; 2008. 283-306.

35. Wu, K.-D., et al., Quantum coherence and state conversion: theory and experiment. npj Quantum Inf, 2020; 6(1): 22.

36. Lloyd, S., Quantum coherence in biological systems. J Phy: Conf Ser, $2011 ; 302$.

37. Tegmark, M., Importance of quantum decoherence in brain processes. Phys Rev E, 2000; 61(4): 4194-4206.

38. Arndt, M., Juffmann, T. and Vedral, V. Quantum physics meets biology. HFSP J, 2009; 3(6): 386-400.

39. Martin-Delgado, M.A. On quantum effects in a theory of biological evolution. Sci Rep, 2012; 2(1): 302.

40. Scholes, G.D., et al. Using coherence to enhance function in chemical and biophysical systems. Nat, 2017; 543(7647): 647-656.

41. Horn, L.J., et al. Coherent Backscatter opposition effect from saturn ring particles and their regoliths. 1996.

42. Nelson, R., et al., Coherent constructive interference in Saturn's rings reported by Cassini VIMS. 2007; 774

43. Nunn, A.V., Guy, G.W. and Bell, J.D. The quantum mitochondrion and optimal health. Biochem Soc Trans, 2016; 44(4): 1101-1110.

44. Hong, C.K. and Mandel, L. Experimental realization of a localized one-photon state. Phys Rev Lett, 1986; 56(1): 58-60.

45. Grangier, P., Roger, G. and Aspect, A. Experimental evidence for a photon anticorrelation effect on a beam splitter: A new light on singlephoton interferences. Europhys Lett (EPL), 1986; 1(4): 173-179.

46. Kim, H., Kwon, O. and Moon, H.S. Experimental interference of uncorrelated photons. Sci Rep, 2019; 9(1): 18375.

47. Scholes, G.D. Quantum-Coherent electronic energy transfer: Did nature think of it first? J Phys Chem Lett, 2010; 1(1): 2-8.

48. Terhal, B.M., Wolf, M.M. and Doherty, A.C. Quantum entanglement: A modern perspective. Phys Today, 2003; 56(4): 46-52.

Nordén, B. Quantum entanglement: Facts and fiction - how wrong was Einstein after all? Q Rev Biophys, 2016; 49: e17.

50. Mooney, G.J., Hill, C.D. and Hollenberg, L.C.L. Entanglement in a 20-Qubit superconducting quantum computer. Sci Rep, 2019; 9(1): 13465 .

51. Wiseman, H. Physics: Bell's theorem still reverberates. Nat, 2014;
510(7506): 467-469

52. Aspect, A. P. Grangier, and G. Roger, Experimental realization of Einstein-Podolsky-Rosen-Bohm Gedanken experiment: A new violation of Bell's inequalities. Phys Rev Lett, 1982; 49(2): 91-94.

53. Moreau, P.-A., et al. Imaging Bell-type nonlocal behavior. Sci Adv, 2019; 5(7): eaaw2563.

54. Tarlaci, S., Quantum physics in living matter: From quantum biology to quantum neurobiology. NeuroQuantology, 2011; 9: 692-701.

55. Adams, B. and Petruccione, F. Quantum effects in the brain: A review. AVS Quantum Sci, 2020; 2(2).

56. Devault, D. Quantum mechanical tunnelling in biological systems. Q Rev Biophys, 1980; 13(4): 387-564.

57. Moser, C.C., Anderson, J.L.R. and Dutton, P.L. Guidelines for tunneling in enzymes. Biochim Biophys Acta, Bioenerg, 2010; 1797(9): 1573-1586.

58. Streltsov, A., et al. Measuring quantum coherence with entanglement Phys RevLett, 2015; 115(2): 020403.

59. Fröhlich, H. Long-range coherence and energy storage in biological systems. Int J Quantum Chem, 1968; 2(5): 641-649.

60. Vattay, G., Kauffman, S. and Niiranen, S. Quantum biology on the edge of quantum chaos. PLoS One, 2014; 9(3): e89017.

61. Lovley, D.R. and Malvankar, N.S. Seeing is believing: nove imaging techniques help clarify microbial nanowire structure and function. Environ Microbiol, 2015; 17(7): 2209-2215.

62. Cai, J. and Plenio, M.B. Chemical compass model for avian magnetoreception as a quantum coherent device. Physical Review Lett, 2013; 111(23): 230503.

63. Liaofu, L. and Guochen, Y. A new model on DNA structure and thermal denaturation. bioRxiv, 2018; 386524.

64. Yang, Z., et al. Revealing quantum mechanical effects in enzyme catalysis with large-scale electronic structure simulation. React Chem Eng, 2019; 4(2): 298-315.

65. Jedlicka, P. Revisiting the quantum brain hypothesis: Toward quantum (Neuro)biology? Front Mol Meurosci, 2017; 10: 366-366.

66. Melkikh, A.V. and Khrennikov, A. Nontrivial quantum and quantumlike effects in biosystems: Unsolved questions and paradoxes. Prog Biophys Mol Biol, 2015; 119(2): 137-161.

67. Pray, L. Discovery of DNA structure and function: Watson and Crick. Nat Educ, 2008; 1(1): 100

68. Cobb, M. Oswald Avery, DNA, and the transformation of biology. Curr Biol, 2014; 24(2): R55-R60.

69. Home, D. and Chattopadhyaya, R. DNA molecular cousin of Schrödinger's cat: A curious example of quantum measurement. Phys Rev Lett, 1996; 76(16): 2836-2839.

70. Mihelic, F.M. Model of biological quantum logic in DNA. Life (Basel, Switzerland), 2013; 3(3): 474-481.

71. Cooper, W.G. Accuracy in biological information technology involves enzymatic quantum processing and entanglement of decohered isomers. Inf, 2011; 2(1): 166-194.

72. Téoule, R. Radiation-induced DNA damage and its repair. Int J Radiat Biol Relat Stud Phys Chem Med, 1987; 51(4): 573-589.

73. Rastogi, R.P., et al. Molecular mechanisms of ultraviolet radiationinduced DNA damage and repair. J Nucleic Acids, 2010; 2010: 592980

74. Schär, P. Spontaneous DNA damage, genome instability, and cancer-when DNA replication escapes control. Cell, 2001; 104(3): 329332.

75. Preston, B.D., Albertson, T.M. and Herr, A.J. DNA replication fidelity and cancer. Semin Cancer Biol, 2010; 20(5): 281-293

76. Singh, V., Fedeles, B.I. and Essigmann, J.M. Role of tautomerism in RNA biochemistry. RNA (New York, N.Y.), 2015; 21(1): 1-13.

77. Cairns, J., Overbaugh, J. and Miller, S. The origin of mutants. Nat, 1988; 335(6186): 142-145.

78. Cooper, W.G. The molecular clock in terms of quantum information processing of coherent states, entanglement and replication of evolutionarily selected decohered isomers. Interdiscip Sci, 2011; 3(2): 91-109.

79. Djordjevic, I.B., Markov chain-like quantum biological modeling of mutations, aging, and evolution. Life (Basel), 2015; 5(3): 15181538 .

80. Chua, E.G., et al. Quantum changes in Helicobacter pylori gene expression accompany host-adaptation. DNA Res, 2017; 24(1): 37 49.

81. Riesenfeld, C., et al. Adaptive mutations produce resistance to ciprofloxacin. Antimicrob Agents Chemother, 1997; 41(9): 2059.

82. Perfeito, L., et al. Adaptive mutations in bacteria: High rate and small effects. Sci, 2007; 317(5839): 813.

83. McKenzie, G. and Rosenberg, S. Adaptive mutations, mutator DNA polymerases and genetic change strategies of pathogens. Curr Opin Microbiol, 2001; 4(5): 586-594.

84. Mickiewicz, K.M., et al. Possible role of L-form switching in recurrent urinary tract infection. Nat Commun, 2019; 10(1): 4379.

85. Svensson, E.I. and Berger, D. The role of mutation bias in adaptive evolution. Trends Ecol Evol, 2019; 34(5): 422-434.

86. Raghunandan, R., et al. A review of applications of principles of quantum physics in oncology: do quantum physics principles have any role in oncology research and applications? J Radiother Pract, 2019; 18(4): 383-394.

87. Laster, M., et al. When cancer meets quantum mechanics. Theor Biol Forum, 2019; 112(1-2): 35-51.

88. Uthamacumaran, A. A biophysical approach to cancer dynamics: Quantum chaos and energy turbulence. Biosystems, 2017; 156-157: $1-22$. 
89. Friedman, R., Boye, K. and Flatmark, K. Molecular modelling and simulations in cancer research. Biochim Biophys Acta, 2013; 1836(1): $1-14$.

90. Djordjevic, I.B. Quantum biological channel modeling and capacity calculation. Life (Basel), 2012; 2(4): 377-391.

91. Bassan, P., et al. Large scale infrared imaging of tissue micro arrays (TMAs) using a tunable Quantum Cascade Laser (QCL) based microscope. Analyst, 2014; 139(16): 3856-3859.

92. Biava, P.M., et al. stem cell differentiation stage factors and their role in triggering symmetry breaking processes during cancer development: A quantum field theory model for reprogramming cancer cells to healthy phenotypes. Curr Med Chem, 2019. 26(6): 988-1001.

93. Yao, J., et al. Biochemistry and biomedicine of quantum dots: From biodetection to bioimaging, drug discovery, diagnostics, and therapy. Acta Biomater, 2018; 74: 36-55.

94. Thakur, M., Kumawat, M.K. and Srivastava, R. Multifunctional graphene quantum dots for combined photothermal and photodynamic therapy coupled with cancer cell tracking applications. RSC Advances, 2017; 7(9): 5251-5261.

95. Luo, G., et al. Quantum dots in cancer therapy. Expert Opin Drug Deliv, 2012; 9(1): 47-58

96. Gao, X., et al. In vivo cancer targeting and imaging with semiconductor quantum dots. Nat Biotechnol, 2004; 22(8): 969-976.

97. I Jacobson, J., A quantum theory of disease, including cancer and the aging process. Fractal Geom Nonlinear Anal Med Biol, 2016; 1(4).

98. Formenti, S.C., et al. Radiotherapy induces responses of lung cancer to CTLA-4 blockade. Nat Med, 2018; 24(12): 1845-1851.

99. Liu, Y., et al. Abscopal effect of radiotherapy combined with immune checkpoint inhibitors. J Hematol Oncol, 2018; 11(1): 104.

100. Siva, S., et al. Abscopal effects of radiation therapy: a clinical review for the radiobiologist. Cancer Lett, 2015; 356(1): 82-90.

101. Reynders, K., et al. The abscopal effect of local radiotherapy: Using immunotherapy to make a rare event clinically relevant. Cancer Treat Rev, 2015; 41(6): 503-510.

102. Courtney, C.M., et al. Photoexcited quantum dots for killing multidrug-resistant bacteria. Nat Materials, 2016; 15(5): 529-534.

103. Bennington-Castro, J., Bio Focus: Light-activated quantum dots kill antibiotic-resistant superbugs. MRS Bulletin, 2016; 41(3): 178-179.

104. Levy, M., et al. Assessing different reactive oxygen species as potential antibiotics: Selectivity of intracellular superoxide generation using quantum dots. ACS Applied Bio Materials, 2018; 1(2): 529-537.

105. Go, Y.-M. and Jones, D.P. Redox theory of aging: Implications for health and disease. Clin Sci (London, England : 1979), 2017; 131(14): $1669-1688$.

106. Franco, R. and Vargas, M.R. Redox biology in neurological function, dysfunction, and aging. Antioxid Redox Signaling, 2018; 28(18): $1583-1586$.

107. Focusing on mitochondrial form and function. Nat Cell Biol, 2018; 20(7): 735.

108. Punekar, N.S. Enzymatic Oxidation-Reduction Reactions. In: ENZYMES: Catalysis, Kinetics and Mechanisms. 2018. 385-402.

109. Gautheron, D.C., Mitochondrial oxidative phosphorylation and respiratory chain: Review. Jnherited Metab Dis, 1984; 7(1): 57-61.

110. Haydon, N., McGlynn, S.E. and Robus, O. Speculation on quantum mechanics and the operation of life giving catalysts. Orig Life Evol Biosph, 2011; 41(1): 35-50.

111. Xin, H., et al. Quantum biological tunnel junction for electron transfer imaging in live cells. Nature Commun, 2019; 10(1): 3245 .

112. Milenkovic, D., et al., The enigma of the respiratory chain supercomplex. Cell Metab, 2017; 25(4): 765-776.

113. Leonard, J.V. and Schapira, A.H.V. Mitochondrial respiratory chain disorders II: neurodegenerative disorders and nuclear gene defects. The Lancet, 2000; 355(9201): 389-394.

114. de Lonlay, P., et al. A mutant mitochondrial respiratory chain assembly protein causes complex III deficiency in patients with tubulopathy, encephalopathy and liver failure. Nat Genet, 2001; 29(1): 57-60.

115. Calvo, S.E. and Mootha, V.K. The mitochondrial proteome and human disease. Annu Rev Genomics Hum Genet, 2010; 11(1): 25-44.

116. Schapira, A.H.V., Primary and secondary defects of the mitochondrial respiratory chain. J Inherited Metab Dis, 2002; 25(3): 207-214.

117. Pokorný, J., et al. Mitochondrial dysfunction and disturbed coherence: Gate to cancer. Pharm (Basel, Switzerland), 2015; 8(4): 675-695.

118. Chin, A.W., Huelga, S.F. and Plenio, M.B. Coherence and decoherence in biological systems: principles of noise-assisted transport and the origin of long-lived coherences. Philos Trans A Math Phys Eng Sci, 2012; 370(1972): 3638-3657.

119. Wang, X., He, X. and Zhang, J.Z. Accurate calculation of electric fields inside enzymes. Methods Enzymol, 2016; 578: 45-72.

120. Hoehn, R.D., et al. Neuroreceptor activation by vibration-assisted tunneling. Sci Rep, 2015; 5: 9990.

121. Hoehn, R.D., et al. Status of the vibrational theory of olfaction. Front Phys, 2018; 6(25)

122. Franco, M.I., et al. Molecular vibration-sensing component in Drosophila melanogaster olfaction. Proc Nat Acad Sci, 2011; 108(9): 3797.

123. Vennemann, M.M., Hummel, T. and Berger, K. The association between smoking and smell and taste impairment in the general population. J Neurol, 2008; 255(8): 1121-1126.

124. Brämerson, A., et al., Prevalence of olfactory dysfunction: The skövde population-based study. The Laryngoscope, 2004; 114: 733-737.

125. Murphy, C., et al. Prevalence of olfactory impairment in older adults. Jama, 2002; 288(18): 2307-2312.

126. Nordin, S. and Bramerson, A. Complaints of olfactory disorders: epidemiology, assessment and clinical implications. Curr Opin Allergy Clin Immunol, 2008; 8(1): 10-15.

127. Damm, M., et al. Olfactory dysfunctions. Epidemiology and therapy in Germany, Austria and Switzerland. HNO, 2004; 52(2): 112-120.

128. Croy, I., S. Nordin, and Hummel, T. Olfactory disorders and quality of life-An updated review. Chem Senses, 2014; 39(3): 185-194.

129. Fanelli, M.T. and Stevenhagen, K.J. Characterizing consumption patterns by food frequency methods: core foods and variety of foods in diets of older Americans. J Am Diet Assoc, 1985; 85(12): 1570-1576.

130. Wysocki, C.J. and Pelchat, M.L. The effects of aging on the human sense of smell and its relationship to food choice. Crit Rev Food Sci Nutr, 1993; 33(1): 63-82.

131. Mattes, R.D. and Cowart, B.J. Dietary assessment of patients with chemosensotyr disorders. J Am Diet Assoc, 1994; 94(1): 50-56.

132. Griep, M.I., et al. Food odor thresholds in relation to age, nutritional, and health status. The J Gerontol: Ser A, 1995; 50A(6): B407-B414.

133. Wilson, M.M., et al. Appetite assessment: simple appetite questionnaire predicts weight loss in community-dwelling adults and nursing home residents. Am J Clin Nutr, 2005; 82(5): 1074-1081.

134. Karpa, M.J., et al. Prevalence and Neurodegenerative or other associations with olfactory impairment in an older community. J Aging Health, 2010; 22(2): 154-168.

135. Barjas Qaswal, A. The myelin sheath maintains the spatiotemporal fidelity of action potentials by eliminating the effect of quantum tunneling of potassium ions through the closed channels of the neuronal membrane. Quantum Rep, 2019; 1(2): 287-294.

136. Bernroider, G. and Summhammer, J. Can quantum entanglement between ion transition states effect action potential initiation? Cognit Comput, 2012; 4(1): 29-37.

137. Moradi, N., F. Scholkmann, and Salari, V. A study of quantum mechanical probabilities in the classical Hodgkin-Huxley model. J Integr Neurosci, 2015; 14(01): 1-17.

138. Gonzalez-Raya, T., et al. Quantized Single-Ion-Channel HodgkinHuxley model for quantum neurons. Phys Rev Appl, 2019; 12(1): 014037.

139. Penrose, R., Shadows of the mind: A search for the missing science of consciousness. Oxford University Press, Inc; 1994.

140. Khrennikov, A., et al. Quantum probability in decision making from quantum information representation of neuronal states. Sci Rep, 2018; 8(1): 16225 .

141. Baars, B.J. and Edelman, D.B. Consciousness, biology and quantum hypotheses. Phys Life Rev, 2012; 9(3): 285-294.

142. Hagan, S., S.R. Hameroff, and Tuszyński, J.A. Quantum computation in brain microtubules: Decoherence and biological feasibility. Phys Rev E, 2002; 65(6): 061901 .

143. Schmidt, H. Quantum $\square$ mechanical random $\square$ number generator. J Appl Phys, 1970; 41(2): 462-468.

144. Dunne, B.J. and Jahn, R.G. Consciousness, information, and living systems. Cell Mol Biol (Noisy-le-grand), 2005; 51(7): 703-714.

145. Radin, D., et al., Consciousness and the double-slit interference pattern: Six experiments. Phys Essays, 2012; 25(2): 157-171.

146. Shi, L., E.J. Galvez, and Alfano, R.R. Photon entanglement through brain tissue. Sci Rep, 2016; 6(1): 37714.

147. Kandori, H., Y. Shichida, and Yoshizawa, T. Photoisomerization in rhodopsin. Biochem (Mosc), 2001; 66(11): 1197-209.

148. Lakhno, V.D., et al. Quantum-classical model of retinal photoisomerization reaction in visual pigment rhodopsin. Dokl Biochem Biophys, 2016; 471(1): 435-439.

149. Shigaev, A.S., et al. Quantum-classical model of the rhodopsin retinal chromophore cis-trans photoisomerization with modified intersubsystem coupling. Comput Theor Chem, 2020; 1181: 112831.

150. Hahn, S. and G. Stock, Quantum-Mechanical Modeling of the Femtosecond Isomerization in Rhodopsin. J Phys Chem B, 2000; 104(6): 1146-1149.

151. Nogly, P., et al. Retinal isomerization in bacteriorhodopsin captured by a femtosecond x-ray laser. Sci, 2018; 361(6398).

152. Prokhorenko, V.I., et al. Coherent control of retinal isomerization in bacteriorhodopsin. Sci, 2006; 313(5791): 1257-1261.

153. Trevors, J.T. and Masson, L. Quantum microbiology. Curr Issues Mol Biol, 2011; 13(2): 43-50.

154. Drakova, D. and Doyen, G. How can the green sulfur bacteria use quantum computing for light harvesting? arXiv e-prints, 2016. arXiv:1605.06149

155. Sarovar, M., et al. Quantum entanglement in photosynthetic lightharvesting complexes. Nature Physics, 2010; 6(6): 462-467.

156. Farrow, T. and Vedral, V. Scale-estimation of quantum coherent energy transport in multiple-minima systems. Sci Rep, 2014; 4(1): 5520.

157. Panitchayangkoon, G., et al. Long-lived quantum coherence in photosynthetic complexes at physiological temperature. Proc Nat Acad Sci, 2010; 107: 12766.

158. Collini, E., et al. Coherently wired light-harvesting in photosynthetic marine algae at ambient temperature. Nature, 2010; 463(7281): 644647.

159. Karafyllidis, I.G. Quantum transport in the FMO photosynthetic lightharvesting complex. J Biol Phys, 2017; 43(2): 239-245.

160. Hoyer, S., M. Sarovar, and Whaley, K.B. Limits of quantum speedup in photosynthetic light harvesting. New J Phys, 2010; 12(6): 065041.

161. Lee, A.A., et al. Alternative radical pairs for cryptochrome-based magnetoreception. J R Soc Interface, 2014; 11(95): 20131063.

162. Hiscock, H.G., et al. The quantum needle of the avian magnetic compass. Proc Nat Acad Sci, 2016; 113(17): 4634

163. Rodgers, C.T. and Hore P.J. Chemical magnetoreception in birds: The radical pair mechanism. Proc Nat Acad Sci, 2009; 106(2): 353. 
164. Fay, T.P., et al. How quantum is radical pair magnetoreception? Faraday Discuss, 2019; 221(0): 77-91.

165. Schulten, K., C.E. Swenberg, and A. Weller. A biomagnetic sensory mechanism based on magnetic field modulated coherent electron spin motion. Zeitschrift für Physikalische Chemie, 1978; 111(1): 1-5.

166. Nießner, C. and M. Winklhofer. Radical-pair-based magnetoreception in birds: Radio-frequency experiments and the role of cryptochrome. J Comp Physiol. A, Neuroethology, sensory, neural, and behavioral physiology, 2017; 203(6-7): 499-507.

167. Maeda, K., et al. Magnetically sensitive light-induced reactions in cryptochrome are consistent with its proposed role as a magnetoreceptor. Proc Nat Acad Sci, 2012; 109(13): 4774.

168. Solov'yov, I., D. Chandler, and K. Schulten, Exploring the possibilities for radical pair effects in cryptochrome. Plant Signaling Behav, 2008 3: 676-677.

169. Baskar, R. et al. Cancer and radiation therapy: Current advances and future directions. Int J Med Sci, 2012; 9(3): 193-199.

170. Vatansever, F. and M.R. Hamblin. Far infrared radiation (FIR): Its biological effects and medical applications. Photonics Lasers Med, 2012; 4: 255-266

171. Grimes, D.R., Ultraviolet radiation therapy and UVR dose models. Med Phys, 2015; 42(1): 440-455.

172. Sultan, L.R. and C.M. Sehgal. A Review of early experience in lung ultrasound in the diagnosis and management of COVID-19. Ultrasound in Medicine \& Biology, 2020; 46(9): 2530²545.

173. Antùnez-Montes, O.Y., D. Buonsenso, and S.O. Paz-Ortega. Rationale for the routine application of lung ultrasound in the management of coronavirus disease 2019 (COVID-19) patients in middle- to lowincome countries. Ultrasound Med Biol, 2020; 46(9): 2572-2574.

174. Pansarasa, O., et al. SOD1 in amyotrophic lateral sclerosis: "Ambivalent" Behavior connected to the disease. Int J Mol Sci, 2018; 19(5): 1345

175. Srinivasan, E. and R. Rajasekaran. Quantum chemical and molecular mechanics studies on the assessment of interactions between resveratrol and mutant SOD1 (G93A) protein. J Comput Aided Mol Des, 2018; 32(12): 1347-1361.

176. Chan, P.K., et al. Structural similarity of wild-type and ALS-mutant superoxide dismutase-1 fibrils using limited proteolysis and atomic force microscopy. Proc Nat Acad Sci, 2013; 110(27): 10934-10939.

177. Drici, N. and M.A. Krallafa. Effect of mutation on the stabilization energy of HIV-1 zinc fingers: a hybrid local self-consistent field/ molecular mechanics investigation. J Biol Inorg Chem, 2017; 22(1): 109-119.

178. Tahan, A. and M. Monajjemi. Solvent dielectric effect and side chain mutation on the structural stability of Burkholderia cepacia lipase active site: a quantum mechanical/molecular mechanics study. Acta Biotheor, 2011; 59(3-4): 291-312. 